\title{
Map3k2-Regulated Intestinal Stromal Cells (MRISC) Define a Distinct Sub-cryptic Stem Cell Niche for Damage Induced Wnt Agonist R-spondin1 Production
}

Ningbo $\mathrm{Wu}^{1,2^{*}}$, Hongxiang Sun ${ }^{1,2^{*}}$, Xiaoyun Zhao ${ }^{1,2}$, Lei Chen ${ }^{1,2}$, Yuanyuan $\mathrm{Qi}^{1}$, Yuheng Han $^{1,2}$, Xianan $\mathrm{Liu}^{1}$, Caixia Gao ${ }^{1}$, Qun $\mathrm{Wang}^{1}$, Lingjuan $\mathrm{He}^{3}$, Xiaoyin $\mathrm{Niu}^{1}$, Zhiduo Liu ${ }^{1}, \mathrm{Hua}-\mathrm{Bing}^{1}{ }^{1,2}$, Yi Arial Zeng $^{3}$, Manolis Roulis ${ }^{4}$, Dou Liu ${ }^{4}$, Zhengfeng Yang ${ }^{1,2}$, Bin Zhou ${ }^{3}$, Richard A. Flavell ${ }^{2,4}, \&$ Bing Su, ${ }^{1,2 \#}$

${ }^{1}$ Shanghai Institute of Immunology, Department of Immunology and Microbiology, and the Minister of Education Key Laboratory of Cell Death and Differentiation, Shanghai JiaoTong University School of Medicine, Shanghai 200025, China.

${ }^{2}$ Yale Institute for Immune Metabolism, Shanghai JiaoTong University School of Medicine, Shanghai 200025, China.

${ }^{3}$ The State Key Laboratory of Cell Biology, CAS Center for Excellence in Molecular Cell Science, Shanghai Institute of Biochemistry and Cell Biology, Chinese Academy of Sciences, University of Chinese Academy of Sciences, Shanghai 200031, China.

${ }^{4}$ Department of Immunobiology, Howard Hughes Medical Institute, Yale University School of Medicine, New Haven, CT 06520, USA.

${ }^{\#}$ Correspondence should be addressed to: bingsu@

*These authors contributed equally to this work. 


\section{Highlights}

1) Map3k2 protects mice from DSS-induced colitis by promoting intestinal stem cell regeneration.

2) Map3k2-MAPK pathway cross-talks with Wnt signaling pathway via upregulation of R-spondin1.

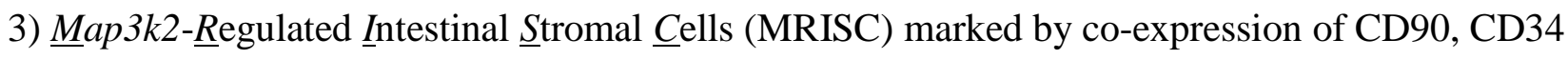
and CD81 defines a novel colonic stem cell niche. 


\section{Summary}

Intestinal stem cell propagation and differentiation are essential for rapid repair of tissue damage in the gut. While intestinal stromal cells were recently identified as key mediators of this process, the cellular and molecular mechanisms by which this diverse population induces tissue repair remains poorly understood. Here we show that Map3k2 has a colon stromal cell specific function critically required for maintenance of $\mathrm{Lgr}^{+}$intestinal stem cells and protection against acute intestinal damage. This Map3k2-specific function is mediated by enhancing Wnt agonist Rspondin1 production. We further reveal a unique novel cell population, named Map3k2-regulated intestinal stromal cells (MRISC), as the primary cellular source of R-spondin1 following intestinal injury. Together, our data identify a novel intestinal stem cell niche organized by MRISC, which specifically dependent on the Map3k2-signaling pathway to augment the production of Wnt agonist R-spondin 1 and promote regeneration of the acutely damaged intestine. 


\section{Introduction}

Stem cell proliferation and differentiation are critical for rapid repair of intestinal injury and maintenance of host health (Nanki et al., 2018). Intestinal stem cells (ISC) are thought to rely on specific signals and growth factors produced by the local niche that support their repair functions and self-renewal in the crypts (Aoki et al., 2016; Degirmenci et al., 2018; Greicius et al., 2018; Kabiri et al., 2014; Sato et al., 2011; Shoshkes-Carmel et al., 2018). In particular, diverse populations of intestinal mesenchymal stromal cells (IMSC) appear to play key roles in ISC niche maintenance via sensing of factors including FGF, TGF- $\beta$, Hedgehog, and PDGF (Koch, 2017; Roulis and Flavell, 2016; Valenta et al., 2016), but the specific IMSC subsets and molecular mediators involved are unknown.

Several studies have now suggested that $\mathrm{CD}^{+} 0^{+}$IMSC may be crucial regulators of gut homeostasis (Huynh et al., 2016; Karpus et al., 2019; Kinchen et al., 2018; Powell et al., 2011; Roulis et al., 2014). However, recent efforts to understand the heterogeneity and function of ISMC using single-cell RNA sequencing (scRNA-seq) and lineage tracing techniques have revealed the existence of multiple functionally distinct subsets (Kinchen et al., 2018; Nanki et al., 2018), many of which produce soluble factors and cytokines likely capable of modulating gut homeostasis and epithelial integrity (Han et al., 2018; Kinchen et al., 2018; Shoshkes-Carmel et al., 2018; Thomson et al., 2018). In particular, IMSC expressing SOX6, CD142 and Wnt pathway genes appear to localize to intestinal crypts and can support epithelial stem cell function(Kinchen et al., 2018), while a population of GLI1-expressing IMSC localized in the pericrypt area has also been shown to mediate colonic stem cell renewal in a murine model (Degirmenci et al., 2018). Similarly two separate populations of fibroblast-like stromal cells (expressing $\mathrm{Ackr} 4^{+} / \mathrm{CD} 34^{+}$or $\mathrm{Foxl1}^{+} / \mathrm{F}^{+}$) in the pericrypt region have been reported to drive stem cell proliferation and gut organoid growth in vitro, likely via production of ligands/agonists of the Wnt and BMP pathways (Aoki et al., 2016; Shoshkes-Carmel et al., 2018; Stzepourginski et al., 2017; Thomson et al., 2018). However, it is currently unclear to what extent these different IMSC populations exert redundant functions or play different roles in gut tissue health and disease states.

The Map3k2 gene encodes a serine/threonine protein kinase belonging to the MAP3K 
superfamily (Cheng et al., 2000; Guo et al., 2002; Su et al., 2001; Sun et al., 2001; Zhang et al., 2006). Map3k2 signaling is triggered by a range of soluble factors including FGF, PDGF, TGF- $\beta$, TNF- $\alpha$ and IL-1 $\beta$, leading to activation of downstream effectors ERK1/2, JNK, p38 and ERK5 via their respective MAPK kinases (Chang et al., 2011; Cheng et al., 2005; Greenblatt et al., 2016; Sun et al., 2001; Tsioumpekou et al., 2016; Zhang et al., 2006). Map3k2 is constitutively expressed in multiple mouse organs including various cell types that are resident in the gut (Guo et al., 2002; Han et al., 2018).

In this report, we used scRNA-seq in combination with flow-cytometry and qRT-PCR to identify a novel population of $\mathrm{CD} 81^{+}$sub-crypt intestinal stromal cells that depend on a specific MAP3K2 signaling cascade to augment expression of Wnt agonist Rspol. We further demonstrate that this signaling axis is critically required for rapid regeneration of damaged intestine via induction of epithelial stem cell proliferation and survival in the colon, leading to efficient host protection against DSS-induced colitis. Together, our data uncover a novel population of stromal cells that maintains an epithelial stem cell niche capable of efficient regeneration of the acutely damaged intestine. 


\section{Results}

\section{Map3k2 Protects Mice against DSS-Induced Colitis}

Map3k2 is expressed in the gut epithelium, stromal and hematopoietic compartments, but germline deficiency of Map3k2 exerts minimal effect on normal mouse growth (Chang et al., 2011; Guo et al., 2002). To investigate the role of Map3k2 in intestinal inflammation, we used an acute colitis model in which co-housed littermates of wild type (WT) and Map3k2/- mice were administered $2 \%$ DSS in their drinking water for 7 days followed by regular drinking water for a further two days thereafter. Within 5-6 days of DSS intake, we observed that Map3k $2^{-/}$mice suffered greater loss of body weight (Figure 1A) and worse diarrhea (Figure 1B) than did WT mice. Endoscopic examination after DSS treatment also revealed more severe bleeding, ulceration, and colonic swelling in Map $3 k 2^{--}$mice than in WT mice (Figure 1C). Furthermore, Map $3 k 2^{--}$mice also exhibited significantly shorter colon length (Figure 1D) as well as more severe epithelial damage and greater leukocyte infiltration than were observed in WT mice on day 9 after DSS treatment (Figure 1E). These data reveal that $M a p 3 k 2$ plays an important role in host protection against acute intestinal tissue damage.

\section{Map3k2 is Required for Intestinal Epithelium Regeneration In Vivo}

Given the more severe colonic damage induced by DSS administration in Map $3 \mathrm{k}^{-/}$mice, we next assessed which specific cell types might be associated with the pathology observed in these animals. While baseline goblet cell number and capacity for mucin production were comparable in colon from both WT and Map3 $\mathrm{k}^{-/-}$mice, after 5 days DSS treatment goblet cell number were substantially reduced and spatial distribution was altered in colonic tissue from Map $3 \mathrm{k} 2^{-/}$animals (Figure 1F). These features were even more pronounced by day 7-9, at which point the Map3k $2^{-/-}$ mice almost entirely lacked mucin producing cells in the gut epithelium (Figures 1F and 1G). Consistently, mRNA levels of the goblet cell-specific marker Muc2 were dramatically reduced in Map3k2-deficient colon comparing to WT colon that were subjected to the same DSS treatment (Figure 1H). Ki67 staining also revealed that proliferation of colonic epithelial cells in response to 
DSS treatment was dramatically reduced in Map3 $k 2^{-/-}$mice compared with WT mice (Data not shown). $\operatorname{Reg} 4^{+}$Deep Crypt Secreting (DCS) cells also displayed hallmarks of increased damage in Map3k2-deficient colon (Data not shown). Together, these data demonstrated that multiple lineages of intestinal epithelial cells were severely disrupted in the colon of Map3 $\mathrm{k}^{-/-}$mice, suggesting that this kinase may be critical for stem cell-mediated epithelial regeneration following acute injury.

\section{Map3k2 Plays a Critical Role in Maintaining Intestinal Stem Cell Frequency}

Intestinal stem cell (ISC) proliferation and differentiation are crucial for regeneration of the gut epithelium (Barker et al., 2010). Given the severity of epithelial damage observed in Map3k ${ }^{-/}$ mice after DSS treatment, we next examined whether ISC survival and differentiation were defective in the absence of the Map3k2 enzyme. We therefore examined colonic expression levels of the archetypal ISC genes Lgr5 (Barker et al., 2007), Ascl2 (Schuijers et al., 2015) and Hopx (Takeda et al., 2011) by qRT-PCR, which suggested a greater loss of this cell type in DSS-exposed intestinal tissue from Map3k2-deficient mice relative to WT animals (Figure 2A). To confirm these results, we next established $L g r 5$-EGFP reporter mice both on WT (WT-Lgr5-EGFP) and Map3k2/(Map3k2/- $L g r 5$-EGFP) backgrounds to facilitate better comparison of the number of $L g r 5$-EGFP ${ }^{+}$ cells present both before and after DSS treatment. In the absence of DSS treatment, numbers of Lgr5-EGFP ${ }^{+}$cells were comparable between colons from WT and Map $3 k 2^{-/-}$mice, but DSS-induced damage consistently led to a greater reduction in $\operatorname{Lgr} 5-\mathrm{EGFP}^{+}$stem cells in Map3k2/- animals, whether assessed by flow cytometry (Figures 2B and 2C) or imaging quantification (Figures 2D and 2E). Together, these findings demonstrate that Map3k2 protects mice against acute DSSinduced colitis by maintaining the ISC compartment.

\section{Map3k2 is Dispensable for ISC Proliferation and Differentiation}

The above data suggested that Map3k2 might play a critical role in ISC survival, proliferation and/or differentiation into mature gut epithelial lineages including goblet cells. To investigate this possibility, we isolated intestinal crypts from WT and Map $3 k 2^{--}$mice for ex vivo 
culture (Sato et al., 2009). To our surprise, there was no difference in growth rates between organoids derived from WT and Map $3 k 2^{-/}$animals (Figures 2F-2H). Furthermore, qRT-PCR analyses confirmed there was no difference in expression of hallmark genes of intestinal stem cells, enterocytes, goblet cells, enteroendocrine cells, $\operatorname{Reg} 4^{+}$DCS cells, or tuft cells (Figure 2I). These data suggested that Map3k2 is not intrinsically required for ISC growth, survival and differentiation, hence the defects observed in Map $3 \mathrm{k}^{-/-}$mice in vivo are likely due to impairment of other cell types surrounding the stem cells and enterocytes.

\section{Map3k2 Expression in Hematopoietic Cells is Not Required for Protection Against Colitis}

Multiple hematopoietic and non-hematopoietic lineages are required to protect the gut against tissue damage. Since Map3k2 is ubiquitously expressed in almost all types of intestinal cells, we postulated that the severe colonic damage observed in Map3 $\mathrm{k}^{-/}$mice could be due to functional impairment of one or many different cell types in the gut (colonic epithelial cells, stromal cells, or hematopoietic leukocytes). We therefore performed a series of bone marrow transfers, using cells from either WT or Map $3 k 2^{-/}$donor mice to reconstitute lethally irradiated WT or Map $3 k 2^{-/-}$ recipient animals prior to induction of DSS colitis. Severity of inflammation was comparable in WT recipient mice irrespective of whether the bone marrow grafts were derived from WT or Map3k2deficient donors, as measured by loss of body weight (Figure S1A), diarrhea severity score (Figure S1B), colon length (Figure S1C), or epithelial integrity (Figure S1D). We were also unable to detect any difference in Lgr5 or Gob5 expression in DSS-treated intestine from WT mice that had received grafts of either type (Figure S1E), suggesting that expression of Map3k2 in hematopoietic cells was not required for protection against acute tissue damage. These findings therefore indicated that Map3k2 expression in non-hematopoietic epithelium or tissue stroma may be critical for tissue repair after DSS-induced injury. Indeed, transfer of WT donor bone marrow into Map $3 k 2^{--}$recipient mice was unable to rescue these animals from severe colitis (Figures S1F-S1I), and lack of Map3k2 function only in non-hematopoietic cells was sufficient to replicate the loss of colonic epithelial stem cells and goblet cells conferred by global gene deletion (Figure S1J). 


\section{Map3k2 is Essential for Wnt Signaling in the Colon}

To better understand the molecular basis of Map3k2-mediated protection against DSSinduced colitis, we next isolated and sequenced total RNA from the colons of WT and Map3k2/mice after one day exposure to DSS or normal drinking water. In order to identify key Map3k2 target genes and associated signaling pathways, genes that were differentially expressed between the paired samples were determined using Gene Set Enrichment Analysis (GSEA). Using this approach, we detected significant up-regulation of chemokine genes in Map3k2-deficient colon after DSS exposure, consistent with the increased inflammatory infiltrate observed in these animals, whereas genes involved in MAPK signaling were down-regulated as expected (Data not shown). However, when we assessed expression levels of genes associated with intestinal stem cell survival and maintenance, we observed that $M a p 3 k 2^{-/-}$mice exhibit potent down-regulation of Wnt signaling genes (Figure 3A) but not the Notch or EGF pathways (Figure 3B). Furthermore, while expression of Wnt pathway genes in untreated colon tissue was comparable between WT and Map3k2/animals, DSS exposure was associated with a dramatic down-regulation of key Wnt pathwayassociated genes such as Axin2, Fzd8, Nr4a3 and Rspol in Map3k2-deficient colon (Figures 3A and 3C). Furthermore, the overall levels of the ligands, receptors, and mediators of the Wnt pathway were all down regulated in Map3k2-deficient colon after DSS treatment (Figure 3D), strongly indicating that Map $3 k 2$ critically regulates Wnt signaling in order to protect the colon against DSS-induced epithelial damage.

Among the Wnt-related genes identified above, Rspol was the most significantly downregulated in Map3k2-deficient colon (Figures 3A and 3C), consistent with the reports that Rspol plays an essential role in maintaining the ISC niche (Kim et al., 2005; Ootani et al., 2009; Sato et al., 2009; Zhao et al., 2007; Zhou et al., 2013). Accordingly, we observed that reducing the concentrations of R-spondin1 in the in vitro culture media led to dose-dependent decreases in growth and survival of intestinal organoids (Figures S2A-S2C), strongly suggesting that peri-crypt concentrations of R-spondin1 may be equally critical for proliferation and survival of gut epithelial 
stem cells in vivo.

\section{Recombinant R-spondin1 Rescues Map3k2 $2^{-/-}$Mice from DSS-Induced Severe Colitis}

To determine whether Map3k2 is crucial for R-spondin 1 induction and protection against DSS-induced intestinal damage, we next performed a rescue experiment using human recombinant (rec) R-spondin1. Specifically, Map $3 k 2^{-/}$mice were administered 2\% DSS in drinking water with daily intraperitoneal injection of $5 \mu \mathrm{g}$ recR-spondin 1 or saline-only control. As shown in Figures 3E and 3F, recR-spondin 1 markedly reduced colitis severity in Map $3 k 2^{-/}$mice as indicated by less pronounced weight loss (Figure 3E) and milder symptoms of diarrhea (Figure 3F). Expression of inflammatory cytokines such as Il6 and Tnf $\alpha$ was also dramatically reduced in recR-spondin1treated mice colon tissues (Figure 3G). We further observed a partial rescue of goblet cell numbers as assessed by AB/PAS staining (Figure 3H), together with increased mRNA expression of Lgr5 (Figure 3I). Together, these data strongly suggest that the protective role of Map3k2 in DSSinduced colitis is mediated via up-regulation of Rspol.

\section{Map3k2 Regulates Intestinal Mesenchymal Stromal Cell Induction of R-spondin1}

R-spondin1 is crucial for optimal activation of the Wnt pathway, which plays wellrecognized roles in maintaining gut integrity and repairing acute tissue damage (Binnerts et al., 2007; de Lau et al., 2014; Kim et al., 2005; Kretzschmar and Clevers, 2017; Pinto et al., 2003; Yan et al., 2017). However, the cellular sources of Wnt agonist R-spondin1 in the damaged gut have not been clearly defined. In order to uncover the key cellular source of Map3k2-dependent Rspo1 expression in the DSS-damaged gut, we used flow cytometry to sort CD $326^{+} \mathrm{CD} 45^{-}$intestinal epithelial cells (IECs), CD45 ${ }^{+} \mathrm{CD} 326^{-}$hematopoietic cells $\left(\mathrm{CD}^{+} 5^{+}\right)$, and $\mathrm{CD} 326^{-} \mathrm{CD} 45^{-} \mathrm{CD} 31^{-} \mathrm{gp} 38^{+}$ intestinal mesenchymal stromal cells ( $\mathrm{gp} 38^{+}$stroma) from untreated and DSS-challenged mouse colon (Figure 4A). In addition, we used anti-CD90 antibody to sort the gp $38^{+}$stromal fraction into CD90 ${ }^{+}$IMSC and CD90- IMSC subsets before assessing Rspol expression by qRT-PCR. As predicted, we were unable to detect Rspol expression in sorted IEC, whereas CD $45^{+}$hematopoietic 
cells and CD90- IMSC expressed only low levels of Rspol (Figure 4B). In contrast, CD90 ${ }^{+}$IMSC displayed marked production of Rspol and higher expression of Map3k2 than did any other colonic cell population analyzed (Figures 4B and 4C). Importantly, we further observed that Rspol expression in $\mathrm{CD}^{+} 0^{+} \mathrm{IMSC}$ was significantly up-regulated following DSS treatment (Figure 4D), but only in the presence of functional Map3k2 (Figure 4D). These data demonstrate that CD90 ${ }^{+}$ IMSC are likely the key stromal population responsible for Map3k2-mediated gut tissue repair.

In order to confirm that $\mathrm{CD} 90^{+} \mathrm{IMSC}$ are physiologically important for supporting the growth of intestinal epithelial stem cells, we performed a stroma-organoid co-culture experiment using isolated $\mathrm{CD}^{+} 0^{+} \mathrm{IMSC}$ or $\mathrm{CD}^{-}{ }^{-} \mathrm{IMSC}$ co-cultured together with colonic crypts in the absence of exogenous R-spondin1. Organoids displayed only limited growth when cultured in isolation, whereas co-culture with $\mathrm{CD}^{+} 0^{+} \mathrm{IMSC}$ led to a marked increase in overall size and the percentages of spheroids (Figures S2D and S2E). Intriguingly, CD90- IMSC not only failed to promote growth but actually appeared to inhibit organoid expansion when compared with cultures that lacked any stroma (Figures S2D and S2E). Having already established that Map3k2-deficient CD90 ${ }^{+}$IMSC was poor inducers of R-spondin1, which was essential for growth and survival of intestinal organoids (Figure S2A), we next assessed whether CD90 ${ }^{+}$IMSC were still able to support organoid growth after deletion of the Map3k2 gene. Using the same stroma-organoid co-culture approach, we observed that $\mathrm{CD}^{+} 0^{+}$IMSC from WT mouse colon were potent inducers of colonic organoid growth after DSS exposure, forming more spheroids with greater circumference than could be established by their Map3k2-deficient counterparts (Figures 4E-4G). In contrast, in animals that did not receive DSS, colonic CD90 ${ }^{+} \mathrm{IMSC}$ derived from either WT or Map $3 \mathrm{k}^{-/-}$mice displayed similar capacities to support organoid growth (Figures 4E-4G). All together, these results strongly suggest that the protective function of Map3k2 in the gut is mediated by CD $90^{+}$but not CD90- IMSC.

\section{Map3k2-Regulated CD90 ${ }^{+}$IMSC Are Localized to the Colonic Sub-Crypt}

To determine the anatomical location of Map3k2-regulated CD90 ${ }^{+} \mathrm{IMSC}$ in the gut, we next performed immunofluorescent staining using stromal specific markers together with an anti-CD90 
antibody. As shown in Figure S3A, we observed characteristic CD90 staining of the lamina propria T cells or innate lymphoid cells (ILCs), but also detected a specific population of $\mathrm{CD}^{+} 0^{+} \mathrm{gp} 38^{+}$cells located just below the crypts where LGR5-EGFP+ stem cells were also situated as described in a recent study (Figure S3A) (Karpus et al., 2019). However, despite lacking the epithelial marker CD326 and leukocyte antigen CD45, further analysis of this population revealed a monolayer-like structure that is not typical of mesenchymal stroma, and subsequent whole-mount anti-CD90 staining identified this as a nexus of lymphatic vessels that co-stained for the endothelial marker LYVE1 (Figures S3B and S3C, Video 1 and Video 2). We therefore reverted to flow cytometry in order to perform a systemic analysis of which colonic subsets express CD90. IEC and gp38 stromal cells were found to express little CD90, and only a very small fraction of CD $45^{+}$cells expressed this molecule (likely the T-cells and ILCs; Figures S3D and S3E). Between 30-50\% of blood endothelial cells (BEC) and gp38 ${ }^{+}$IMSC were found to express moderate levels of CD90, whereas high-level expression of this marker was restricted to lymphatic endothelial cells (LEC) (Figure S3E). Indeed, when assessed by flow cytometry, LEC expression levels of CD90 were approximately 10-fold higher than those detected for Map3k2-regulated gp $38^{+}$IMSC (Figure S3E). While we anticipate that $\mathrm{CD}^{+} 0^{+} \mathrm{IMSC}$ are situated in a similar location to LEC in vivo, the levels of CD90 expressed by lymphatic endothelial cells prevented Map3k2-dependent CD90 ${ }^{+}$IMSC from being confidently located by microscopy. Consequently, we hereafter refer to CD $90^{+}$IMSC as 'CD90 medium-high' (CD90 $\left.{ }^{\mathrm{mid}}\right)$ cells.

Although CD90 staining did not reveal the identity of the Map3k2-regulated IMSC, a subcrypt location for these cells could be predicted due to the profound effect of Map $3 k 2$ deletion on the ISC niche after DSS challenge. To examine this possibility, we used the fluorescence in situ hybridization (FISH)-based technique RNAScope to detect Rspol mRNA in tissue sections, which revealed that DSS exposure in drinking water was able to induce Rspol expression in the colonic epithelium of WT mice (Figure 4H). Quantitation of Rspol expression levels showed marked induction in the base areas of the sub-epithelium after DSS-treatment, but this was blunted in colon from Map $3 k 2^{--}$mice (Figure 4I). In contrast, Rspol expression in the middle and top layers of the 
sub-epithelial region was comparable in colons from both WT and Map3k2-deficient animals, irrespective of DSS treatment (Figure 4I). Together, these data suggest that Map3k2-mediated Rspol expression defines a novel ISC niche located at the bottom of the crypts.

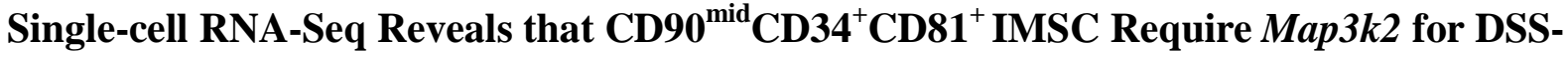 induced Rspo1 Expression}

Having determined that CD90 ${ }^{\text {mid }}$ IMSC are likely the critical subset of Map3k2-regulated cells expressing Rspol in response to acute gut injury, we next sought to uncover the precise features of this stromal population using droplet-based scRNA-seq. First, we sorted CD90 ${ }^{\text {mid }}$ IMSC from DSS-treated colonic lamina propria by gating out hematopoietic cells $\left(\mathrm{CD} 45^{+}\right)$, epithelial cells $\left(\mathrm{CD} 326^{+}\right)$, endothelial cells $\left(\mathrm{CD} 31^{+}\right)$, and gp38 ${ }^{-}$stromal cells (gp38 ) (Figure S4A and S4B), then sequenced 11,116 individual CD90 ${ }^{\text {mid }}$ IMSC using the 10x Genomics platform. Based on unsupervised clustering using Seurat software (Butler et al., 2018), CD90 ${ }^{\text {mid }}$ IMSC were grouped into 10 distinct subsets with specific gene expression profiles (Amir el et al., 2013) (Figure 5A), each of which lacked expression of genes excluded by our cell sorting strategy (CD31, CD45, CD326) (Figure 5B). Preliminary analysis confirmed that CD90 ${ }^{\text {mid }}$ cells were positive for Pdpn (gp38), Vim, Colla2, and Ly6a, but did not express the enteric neuronal cell marker gene Ret (Figure 5B). According to the schemes utilized in other studies (Powell et al., 2011), these 10 IMSC clusters could be tentatively assigned as myofibroblasts (cluster 4) (Mifflin et al., 2011), MHC II stromal cells (cluster 6) (Messina et al., 2017), telocytes (cluster 7) (Shoshkes-Carmel et al., 2018), mesothelial cells (cluster 8) (Rinkevich et al., 2012), interstitial cells of cajal (cluster 9) (Kinchen et al., 2018), contaminating epithelial cells (cluster 10) (Haber et al., 2017), and at least four subgroups of mesenchymal stromal cells for which no prior identifying markers have been reported (clusters 1, 2, 3, \& 5) (Figure 5A). Among these populations, clusters 2, 5, and 8 were found to express Rspol at high levels, identifying these as candidate Map3k2-regulated stromal cells potentially capable of responding to DSS-induced gut tissue damage (Figure 5C). Of these subsets, cluster 8 mesothelial cells are situated away from the crypt region and are thus unlikely to play a 
direct role in supporting epithelial reconstitution. We therefore focused subsequent analyses on the remaining Rspo 1-expressing cell clusters 2 and 5.

To investigate the properties of these two stromal cell populations, we first examined the characteristic marker genes that differentiated these into clusters 2 and 5. Differentially expressed gene analysis indicated that cluster 2 was more pro-inflammatory, as indicated by preferential expression of leukocyte/endothelial cell chemokine $\mathrm{Cxcl14}$ and the activation-linked transcription factor Mafb. In contrast, cluster 5 cells displayed a profile consistent with involvement in tissue remodeling, and preferentially expressed the neuro-regulator Penk, Wnt modulator Sfrp4, and cell development regulator tetraspanin $C d 81$ (Figure 5D). Since both cluster 2 and 5 cell subsets lacked genes such as Sox6 that were characteristic of other niche-associated stromal cells such as telocytes (Shoshkes-Carmel et al., 2018), these data suggest that the populations detected by our analyses represent two previously unknown stromal cell lineages with potential roles in regulating the intestinal stem cell niche (Figure 5D).

Using violin plots to compare specific gene expression within the different cell populations, we found that cluster 2 population expressed mediators typically associated with angiogenesis ( $\mathrm{Cxcl14}$ and $\mathrm{Agt}$ ), whereas cluster 5 cells were found to express genes potentially related to tissue damage and repair (Figf and Ccl7) (Figure 5E). Indeed, cluster 2 cells were further defined by unique expression of the transcription factors Snail and Idl, while cluster 5 cells instead expressed $A r$ and Ebfl (Figure 5E), indicating that these two stromal lineages exert different functions in the colon. In order to facilitate further analysis of these populations by flow cytometry, we next examined their expression profiles of cell-surface markers, which revealed that cluster 5 cells displayed high level of CD81 and CD34 but not CD138 (Sdc1), whereas cluster 2 cells lacked CD81 and CD34 but were CD138 positive (Figure 5F). To determine which of these populations was the major Map3k2-regulated subset involved in gut tissue repair, we next stained colonic lamina propria single cell suspensions with a panel of lineage-defining antibodies (CD326, CD45, CD31, gp38, CD90, CD81, CD34, CD138). The results revealed that about $82.4 \%$ of $\mathrm{CD} 81^{+} \mathrm{CD} 90^{\mathrm{mid}}$ stromal cells, which were fewer than $\sim 4 \%$ of the total gp $38^{+}$stromal cells, were 
likely cluster 5 cells, whereas $10.2 \%$ of $\mathrm{CD} 81^{-} \mathrm{CD} 90^{\mathrm{mid}}$ stromal cells belonged to cluster 2 (Figure 5G). When we sorted these two populations of Rspol ${ }^{+}$IMSC from unchallenged mice, we observed comparably-sized populations in both WT and Map3k2-deficient colon (Figures 5H and 5I). After DSS treatment, we detected a very small increase of cluster 5 cell frequency $\left(\right.$ CD90 ${ }^{\mathrm{mid}}$ $\mathrm{CD} 34^{+} \mathrm{CD} 81^{+} \mathrm{CD} 138^{-}$) in both WT and $M a p 3 k 2^{--}$mice (Figure 5H), whereas cluster 2 cells $\left(\mathrm{CD} 90^{\mathrm{mid}} \mathrm{CD} 81^{-} \mathrm{CD} 34{ }^{-} \mathrm{CD} 138^{+}\right)$appeared unaffected by DSS damage (Figure 5I). Furthermore, basal expression of Rspol gene was similar in cluster 5 cells from both WT and Map3k2/- mice, but after DSS administration expression of Rspol in this cluster was substantially upregulated only in Map3k2-competent cells (Figure 5J). In contrast, cluster 2 cells expressed comparable low levels of Rspol in both WT and Map3k2 animals, and the modest increase in Rspol observed after DSS challenge appeared unaffected by the absence of functional Map3k2 (Figure 5J). Together, these data demonstrate that the use of common lineage markers together with CD81 and CD34 has identified cluster 2 as the Map3k2-regulated stromal cells implicated in ISC niche maintenance.

If Map3k2-dependent $\mathrm{CD} 81{ }^{+} \mathrm{CD} 34^{+}$stromal cells were a genuine niche-forming population, we would expect these cells to be situated in close proximity to intestinal stem cells. Before testing this hypothesis using imaging approaches, we first validated our staining strategy by using flow cytometry to determine which colonic lamina propria populations express both CD81 and CD34. As shown in Figure 5K, IECs displayed only low-level expression of CD81 while completely lacking $\mathrm{CD} 34$, and the few $\mathrm{CD} 45^{+}$leukocytes that stained positive for CD81 were mostly negative for CD34. LECs were consistently negative for both markers. Around 38.8\% of BECs expressed modest levels of CD34 in conjunction with CD81, but these cells could be clearly distinguished from stromal cells based on the additional expression of CD31. Finally, dual expression of CD81 and CD34 was negligible among gp38 stromal cells and totally absent within the CD90` stromal compartment. Taken together, these data confirmed that $\sim 12 \%$ of the $\mathrm{CD} 90^{\mathrm{mid}}$ stromal population could be uniquely defined as $\mathrm{CD} 81^{+} \mathrm{CD} 34^{+}$'double-positive' cells, and could therefore be identified by image analysis for assessment of their tissue distribution within the gut.

We next used antibodies against CD81, CD34 and CD31 to perform immunofluorescence 
analysis of colon sections in order to determine whether $\mathrm{CD} 81^{+} \mathrm{CD} 34^{+}$stromal cells could be identified within the sub-crypt region. While anti-CD81 antibody labelled most cells in colon (red), no staining was observed within the sub-crypt region which was instead dominated by CD34 (green) co-expression with CD31 (blue) indicative of endothelial cells (BEC, LECs) (Figure 5L). However, we were also able to detect CD31 ${ }^{-}$cells that co-expressed CD81 and CD34 (yellow) near the bottom of the crypts, where they appeared to be tightly associated with lymphatic vessels (Figure 5L, Video 3). These results may explain why we were previously unable to visualize these cells using only anti-CD90 staining, since this marker is expressed at 10-fold higher levels by adjacent LECs (Figure S3E). Taken together, these data indicate that a novel lineage of colonic mesenchymal stromal cells defined by co-expression of CD81 and CD34 (but not CD31) is situated close to the lymphatic vessels and depends on Map3k2 to induce Rspo 1 expression following DSS-induced tissue damage. Hereafter, these cells will be referred to as Map3k2-regulated intestinal stromal cells (MRISC).

\section{Conditional Ablation of Map3k2 in Mice Confirms its Intestinal Stromal Cell Specific Roles}

To conclusively demonstrate the stromal cell specific role of Map3k2 in vivo for its protection of damaged colon, we next generated a Map3k2 floxed (Map3k $\left.2^{f l f l}\right)$ mouse line by inserting two loxp sequences flanking the exon 5 of Map3k2 gene (Figure S5A). The tissue specific deletion of Map3k2 gene in these mice was confirmed by first crossing these mice with a T cell specific CD4-Cre line (Sawada et al., 1994). CD4 ${ }^{+}$T cells and CD19 ${ }^{+}$B cells were FACS sorted from control Map3k $2^{f l f l}$ or CD4-Cre:Map3k $2^{f l f l}$ mice for PCR genotyping, and mouse tail DNAs were extracted from WT mice as well as Map $3 k 2^{f l f l}$ and $M a p 3 k 2^{f l /+}$ as controls for the PCR genotyping (Figure S5B). Cell lysates from $\mathrm{CD} 4^{+} \mathrm{T}$ cells and $\mathrm{CD} 19^{+} \mathrm{B}$ cells were further used for immunoblotting to verify the tissue specific Map3k2 deletion in $\mathrm{CD}^{+}{ }^{+} \mathrm{T}$ cells but not $\mathrm{B}$ cells (Figure S5C). These results demonstrate that the Map $3 k 2^{f l f f l}$ mice can be used for tissue specific deletion of the Map3k2 gene. 
We next bred the Map3k $2^{f l f l}$ mice with a commercially available stromal cell specific transgenic line Pdgfra-Cre $e^{E R T}$ to study the in vivo role of Map3k ${ }^{f l f l}$ in the gut (Kang et al., 2010). However, we found out that this particular transgenic Cre line could not delete the target genes from the gut stromal cells effectively although it was reported to delete genes in stromal cells in other organs/tissues (Data not shown). Therefore, we generated a new stromal specific Colla2-Cre ERT2 line, in which a $C r e^{E R T 2}-W P R E$-polyA cassette was inserted in the first exon of Colla2 gene in frame with the translational starting codon between the 5'UTR and the coding sequence (CDS) (Figure S5D and S5E). To confirm that the Colla2-Cre $e^{E R T 2}$ line could lead to specific Map3k2 gene deletion in the colon stromal cells, we sorted the CD $326^{+} \mathrm{IEC}, \mathrm{CD} 45^{+}$leukocytes, CD $31^{+}$ endothelial cells $\left(\mathrm{CD} 45^{-} \mathrm{CD} 326^{-} \mathrm{CD} 31^{+}\right)$, and gp38 ${ }^{+}$intestinal mesenchymal stromal cells (IMSC)

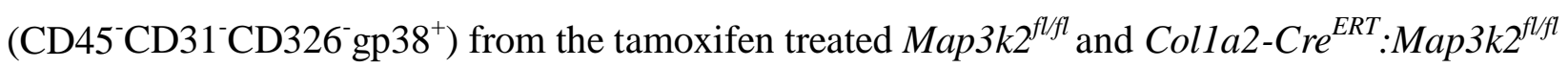
mouse colon, and performed qRT-PCR for the expression of Map3k2. As shown in Figure 6A, we found that Map3k2 expression was efficiently and specifically ablated only in IMSC from the tamoxifen treated Colla2-Cre ${ }^{E R T 2}: M a p 3 k 2^{f l f l}$ mouse colon but not from the control tamoxifen treated Map $3 k 2^{f l f l l}$ mouse colon. Furthermore, Map3k2 expression was not affected in CD $326^{+}$IEC, $\mathrm{CD} 45^{+}$leukocytes, or $\mathrm{CD} 1^{+}$endothelial cells sorted from either the tamoxifen treated control Map3k2 $2^{f l f l}$ or Colla2-Cre $e^{E R T 2}:$ Map3k2 $2^{f l f l}$ mouse colon (Figure 6A). This is consistent with our scRNA-seq data that Colla2 is specifically expressed in colon stromal cells (data not shown). Together these data demonstrate that tamoxifen treatment of Colla2-Cre ${ }^{E R T 2}: M a p 3 k 2^{f l f l}$ mice could achieve stromal specific deletion of Map3k2 in mouse colon.

We next performed DSS-induced colitis experiments with co-housed littermates of Map3k $2^{f l f l}$ and Colla2-Cre $e^{E R T 2}:$ Map3k2 $2^{f l f l}$ mice after tamoxifen treatment. Within 5-6 days of DSS intake, we observed that Colla2-Cre $e^{E R T 2}: M a p 3 k 2^{f l f l}$ mice suffered greater loss of body weight (Figure 6B) and worse diarrhea (Figure 6C) than did Map $3 k 2^{f l f l}$ mice. Colla2-Cre $e^{E R T 2}:$ Map $3 k 2^{f l f l}$ mice also exhibited significantly shorter colon length (Figure 6D) as well as more severe epithelial damage and greater leukocyte infiltration than were observed in Map3k $2^{f l f l}$ mice on day 9 after DSS treatment (Figure 6E). Furthermore, we observed reduced Lgr5 expression and increased Il6 
expression in Colla2-Cre $e^{E R T 2}:$ Map $3 k 2^{f l f l}$ mouse colon as compared to that in Map $3 k 2^{f l f f l}$ mouse colon suggesting more stem cell loss and augmented inflammation (Figure 6F). These data together reveal that Map3k2 plays a stromal specific role in vivo in host protection against acute intestinal tissue damage.

To further rule out the potential role of $M a p 3 k 2$ in intestinal epithelial cells in protection of mice from DSS-induced colitis, we also generated epithelial specific Map3k2 conditional KO mice, by crossing the Map $3 k 2^{f l f l}$ line with Vill-Cre mice, an intestinal epithelial cell specific Cre line (Madison et al., 2002). The expression of Map3k2 was efficiently ablated in purified IEC but not IMSC from the Vil1-Cre:Map3k2 $2^{f l f l}$ mice colon (Figure 6G). Using a similar mouse colitis model described above, we found that Vil1-Cre:Map $3 k 2^{f l f l}$ mice developed similar degree of DSS-induced colitis as that of the control Map $3 k 2^{f l f l}$ mice as measured by body weight loss (Figure 6H), diarrhea scores (Figure 6I), colon length change (Figure 6J), and tissue damage severity (Figure 6K). There was also no significant difference in the stem cell loss or inflammation level in the colons of control Map3k2 $2^{f l f l}$ mice and Vill-Cre:Map3k2 $2^{f l f l}$ mice as measured by Lgr5 and Il6 expression (Figure 6L). Finally, we also generated CD4-Cre:Map3k $2^{f l f l}$ mice to rule out T cell specific role of Map3k2 in protection of mice from DSS induced colitis, and found no significant difference in

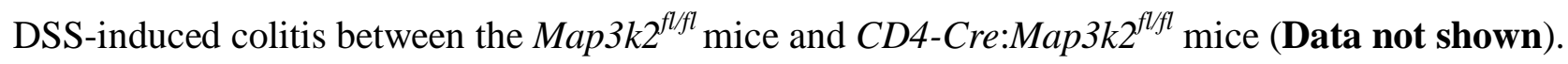

\section{Discussion}

Mesenchymal stromal cells are a heterogeneous population that exerts a range of structural and regulatory functions in the intestine. Multiple subsets of stromal cells have recently been shown to provide critical support for the ISC compartment (Kinchen et al., 2018), but it has remained unclear whether a specific lineage of niche-specific stromal cells regulates ISC proliferation and survival in response to acute tissue damage. In the current study, we identify a novel intestinal population of $\mathrm{CD} 90{ }^{\mathrm{mid}} \mathrm{CD} 81^{+} \mathrm{CD} 34^{+}$mesenchymal stromal cells that are major source of the Wnt agonist Rspolin colon. Anatomically, these Map3k2-regulated intestinal stromal cells (MRISC) are located near the bottom of the colonic crypts, where we propose they are likely to form a unique 
intestinal stem cell niche. The ability of MRISC to augment Rspol expression following colonic injury reflects a likely critical role in sustaining Wnt signaling in intestinal stem cells, leading to rapid epithelial regeneration and repair.

Similar to their roles in other organs and tissues, mesenchymal cells in the intestine are key regulators of epithelial homeostasis, matrix remodeling, immunity, and inflammation (Owens and Simmons, 2013; Pinchuk et al., 2010; Powell et al., 2011). While mesenchymal stromal cells in the gut express a range of common marker genes including Pdpn, Vim, Colla, Collb, Col6a, increasing evidence suggests that there is extensive heterogeneity within this compartment (Kinchen et al., 2018). However, at present there are no known phenotypic markers that allow unique stromal cell subsets to be distinguished within this heterogeneous group. In the current study, our analyses of Map3k2 function in experimental colitis revealed that a specific subset of stromal cells critically depends on this enzyme to mediate damage-induced Rspol expression. Further investigation confirmed that this novel population displayed moderate expression of CD90, which subsequently analysis of this subset by multi-parameter flow cytometry (although lymphatic endothelial cells [LEC] located within the same sub-crypt region express 10-fold higher levels of CD90 that can complicate immunostaining approaches). Specific surface markers that define MRISC were identified using scRNA-seq, which revealed that these stromal cells also express CD81 and CD34 but lack CD31 and CD138. When used in combination, these markers facilitated MRISC visualization without the need for CD90 staining, which allowed subsequent confirmation that

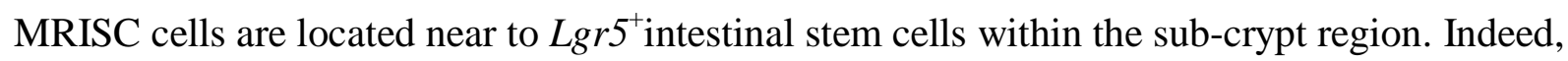
MRISC also appeared to interact with adjacent LECs and local immune cells, suggesting that this lineage may indeed organize multiple cell types into a specific stem cell niche in the gut.

Intriguingly, while our data demonstrate that MRISC are critically important for regeneration and repair of the damaged intestine, these cells, or at least the Map3k2-Rspol axis in these cells, may not be essential for normal gut maintenance or homeostasis. These findings contrast with the recent identification of telocytes and Glil-expressing stromal cells that appear critical for steady-state maintenance of the intestine (Degirmenci et al., 2018; Shoshkes-Carmel et al., 2018). 
Indeed, while telocytes are $\mathrm{Foxll}^{+}$stromal cells that populate the sub-epithelium from stomach to colon, we observed that MRISC are Foxl1 ${ }^{-}$cells with distinct morphology and localization characteristics. Similarly, while Glil-expressing stromal cells (including Foxl1 ${ }^{+}$telocytes) are essential sources of Wnt in the intestine, it is unknown whether these cells are involved in driving colonic Rspol expression and stem cell proliferation in the damaged gut as was demonstrated for MRISC. Based on these differences in gene expression, cell morphology, distribution, and unique responsiveness to tissue damage, we propose that MRISC are a novel population of intestinal stromal cells with important niche functions distinct from either telocytes or Glil-expressing stromal subsets.

Wnt and BMP ligands form a gradient of antagonistic signals in the gut that are known to regulate epithelial renewal and crypt-villi maintenance (Angerer et al., 2016). While the $\mathrm{CD} 11^{+} \mathrm{CD} 90^{\mathrm{mid}} \mathrm{MRISC}$ identified in our study might be predicted to produce Rspol and maintain a steady level of Wnt signaling in the intestinal stem cell niche, little is known about how Wnt signals are regulated following acute intestinal damage. In addition to Rspo1, MRISC were also observed to express Rspo3 and Wnt ligands Wnt2b, Wnt5a and the BMP pathway inhibitors Gremlin1, Gremlin2, further indicating that MRISC may also have a potential role in supporting ISC niche.

Gut microbiome has been shown to be critical for intestinal homeostasis and tissue regeneration (Elinav et al., 2011). It is possible that the Map3k2 mediated protection of gut damage might be affected by commensal bacteria or Map3k2 deficient mice may have a disorder in gut microbiome leading to more severe colitis. However, all our mice were co-housed and treated with DSS together. In addition, we also delivered antibiotics cocktail (Ampicillin, Vancomycin, Neomycin, Metronidazole, and Gentamycin) to either WT or Map3k2 deficient mice before and during colitis induction with DSS, and observed similar results in co-housed mice as those nontreated mice (Data Not Shown). Therefore, the host microbiome is unlikely the major factor for the Map3k2 deficiency caused severe gut damage.

In summary, our study reveals that a novel population of Map3k2-regulated intestinal stromal cells (MRISC) is crucial for induction of Rspol and Wnt signaling which promote rapid 
stem cell proliferation in response to acute intestinal damage. The sub-crypt location of MRISC likely reflects a requirement for direct communication with ISC following tissue injury, although it is currently unclear whether MRISC expression of additional mediators such as IL-6 and IL-33 reflects an ability to interact with other nearby cell types e.g. ILCs, macrophages, and neutrophils. Deeper investigation of the roles played by MRISC in restraining intestinal inflammation and immunopathology will shed important new light on a range of major pathological conditions. In particular, these studies may provide new rationales for use of MAPK inhibitors or Wnt modulators for the treatment of IBD and colitis-associated colorectal cancer.

\section{Acknowledgements}

We would like to thank Drs. Yuan Zhuang, Florent Ginhoux, Jinke Cheng, Yu Li, and Qijun Wang for reading of our manuscript and valuable suggestions. We also want to thank Drs. Zizhen Kang, Honglin Wang for sharing mice. This work was supported in part by grants from the National Natural Science Foundation of China (31470845, 81430033 and 81871269), Shanghai Science and Technology Commission (13JC1404700), Scientific Research Project of Shanghai Municipal Commission of Health and Family Planning (201640137) and the Interdisciplinary Program of Shanghai JiaoTong University (YG2014MS77). 


\section{Figure legends}

\section{Figure 1. Map3k2 Protects Mice from DSS-Induced Colitis}

(A) WT mice $(n=6)$ and Map3 $k 2^{-/}$co-housing littermates $(n=6)$ were administered $2 \%$ DSS in drinking water for 7 days followed by regular drinking water for a further 2 days. In the untreated group, WT mice $(\mathrm{n}=4)$ and $\operatorname{Map} 3 \mathrm{k} 2^{-/}$littermates $(\mathrm{n}=3)$ were provided with regular drinking water for entire the duration of the experiment. Body weights were recorded daily. Shown is a representative graph of three independent experiments.

(B) Daily stool scores for mice treated as described in panel (A).

(C) Representative colonoscopy pictures of WT and Map3k2 $2^{--}$mice before and after 7 days DSS administration in drinking water (left panels) and bar graph quantification of the corresponding pathology scores (right panels) (each $\mathrm{n}=5$ ).

(D) Colon length in mice treated as described in panel (A) before sacrifice on day 9. Bar graphs show colon lengths within each experimental group (right panels).

(E) Hematoxylin and eosin (H\&E) staining of colon (left panels) and histological assessment of the mucosa (right panels) in mice treated as described in panel (A) before sacrifice on day 9. Arrowheads indicate infiltrating leukocytes. Pictures are representative of three independent experiments.

(F) WT and Map3k2 $2^{--}$co-housing littermate mice were administered $2 \%$ DSS in drinking water and then sacrificed on day $0,3,5$, or 7 ( $n=3-4$ mice/time-point). A separate group of mice was subjected to the same regimen for 7 days then transferred to regular drinking water for 2 days prior to sacrifice (Day 9) (n=3). Shown are representative AB/PAS staining of WT and Map3k2 $2^{-/}$mouse colons.

(G) Goblet cell number per crypt in mice treated as described in panel (F) (20 crypts were counted in total from $n=3$ animals per time point).

(H) mRNA expression levels of goblet cell marker gene $M u c 2$ in colon tissue from mice treated as described in panel (F). Data shown are normalized to Hprt expression level. 
Error bars indicate mean $\pm \operatorname{SEM}(* \mathrm{p}<0.05, * * \mathrm{p}<0.01, * * * \mathrm{p}<0.001$ by unpaired Student's $\mathrm{t}$ test $)$.

\section{Figure 2. Map3k2 Maintains Intestinal Stem Cell Frequency Following Tissue Damage}

(A) Lgr5, Ascl2 and Hopx mRNA expression levels in colon tissue from WT and Map3k2 $2^{-}$

littermate mice (each $n=6$ ) after administration of $2 \%$ DSS in drinking water for 5 days. Untreated mice $(n=6)$ were provided with regular drinking water throughout. Data shown are normalized to Hprt and representative of two independent experiments.

(B) Proportions of $\mathrm{EGFP}^{+}$cells within the total $\mathrm{CD}^{-} 5^{-} \mathrm{CD} 326^{+}$(Epcam) colonic epithelial cell compartment as determined by flow cytometry in WT (without GFP), Lgr5-EGFP, and Map $3 k 2^{-/}$Lgr5-EGFP mice $(\mathrm{n}=4)$ after 5 days treatment with $2 \%$ DSS in drinking water (or regular drinking water control; $n=3)$.

(C) Quantitation of $\mathrm{EGFP}^{+}$cells within the total $\mathrm{CD}^{-} 5^{-} \mathrm{CD} 326^{+}$population of colonic epithelial cells from mice treated as described in panel (B).

(D) Lgr5-EGFP and Map3k2 $2^{--}$-Lgr5-EGFP mice were administered 2\% DSS in drinking water for 5 days (or regular drinking water only / untreated). After 5 days, whole colons were dissected for swiss roll sectioning and stained with DAPI for visualization of $\mathrm{GFP}^{+}$crypts using a Leica SP8 microscope. Dashed orange box inserts showed the representative area in each panel.

(E) Quantitation of Lgr5-EGFP ${ }^{+}$crypts in untreated and DSS-challenged mouse colons. The percentage of $\mathrm{EGFP}^{+}$crypts in per 40 crypts were counted $(\mathrm{n}=8)$. Shown are representative pictures from two independent experiments.

(F) Bright-field images of colonic crypts from WT and $M a p 3 k 2^{-/}$mice after $1-5$ days culture in matrigel with Wnt3a, EGF, Noggin and R-spondin1 as indicated. Pictures shown are representative of three independent experiments.

(G) Statistical analysis of average colonoid circumference $(\mathrm{n}=8)$ as derived from WT and Map $3 \mathrm{k} 2^{-/-}$ mice.

(H) Statistical analysis of average colonoid frequency (survival rate) $(n=6)$ as derived from WT and $\operatorname{Map} 3 k 2^{-/-}$mice. 
(I) WT and Map3k2-deficient colonoid mRNA expression level of Lgr5 (intestinal stem cell marker), Alpi (enterocyte marker), Gob5 (goblet cell marker), Chga (enteroendocrine cell marker), Reg4 $\left(\operatorname{Reg} 4^{+}\right.$deep crypt secretory [DCS] cells) and Dclk1 (tuft cell marker) after 5 days culture in matrigel with Wnt3a, EGF, Noggin and R-spondin1 (n=6). Data shown are normalized to Hprt expression level.

Error bars indicate mean \pm SEM $(* * \mathrm{p}<0.01, * * * \mathrm{p}<0.001$ by unpaired Student's $t$ test $)$.

\section{Figure 3. Wnt agonist R-spondin1 Rescues Map3k2-/- Mice from DSS-Induced Severe Colitis}

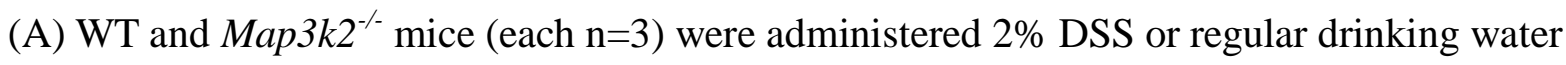
(untreated) for one day before total colon tissue RNA was isolated for RNA-seq. Heat-map of the RNA-seq data showing Wnt pathway genes that were differentially expressed between WT and Map3k2-deficient mouse colons.

(B) Gene Set Enrichment Analysis (GSEA) of the Notch pathway and EGFR response target genes in WT and Map3k2-deficient colons was performed and is presented as an enrichment plot.

(C) qRT-PCR analysis of Axin2, Fzd8, Nr4a3 and Rspol mRNA levels in colonic tissue from mice treated as described in panel (A). Data are normalized to Hprt expression level.

(D) Gene Set Enrichment Analysis of Wnt ligands, Wnt receptors, and Wnt signal mediators in WT and Map3k2 deficient colons from mice treated as described in panel (A).

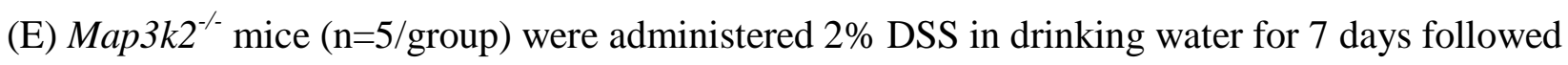
by regular drinking water for 2 days. Human recombinant R-spondin 1 was injected at a daily dose of $5 \mu \mathrm{g} / \mathrm{mouse}$ (from day 1-9). Control mice received saline injection only. Body weight was monitored daily.

(F) Daily stool scores from mice treated as described in panel (E).

(G) Il6 and Tnfa mRNA expression levels in colon tissue from mice treated as described in panel (E) before sacrifice on day 9. Data shown are normalized to Hprt expression level.

(H) Representative Alcian Blue/Periodic Acid Schiff (AB/PAS) staining of colons from mice treated as described in panel (E) before sacrifice on day 9 (left panels). Goblet cell number per crypt is 
shown on the right ( $\mathrm{n}=5 \mathrm{mice} / \mathrm{group}, 30$ crypts counted per group).

(I) Lgr5 mRNA expression in colon tissue from mice treated as described in panel (E) before sacrifice on day 9. Data shown are normalized to Hprt expression level.

Error bars indicate mean \pm SEM $(* \mathrm{p}<0.05, * * \mathrm{p}<0.01, * * * \mathrm{p}<0.001$ by unpaired Student's $\mathrm{t}$ test).

\section{Figure 4. Map3k2-Deficient CD90 ${ }^{+}$Stromal Cells Display Impaired Rspo1 Induction}

(A) Representative flow-cytometry plots showing the percentages of $\mathrm{CD}^{2} 26^{+}$intestinal epithelial cells (IEC), $\mathrm{CD}^{+} 5^{+}$leukocytes, CD90- intestinal mesenchymal stromal cells (CD45 CD31 ${ }^{-} \mathrm{CD}^{-}{ }^{-}$ gp $\left.38^{+} \mathrm{CD} 90^{-}\right)$, and $\mathrm{CD} 90^{+}$intestinal mesenchymal stromal cells $\left(\mathrm{CD} 45^{-} \mathrm{CD} 31^{-} \mathrm{CD} 326^{-} \mathrm{gp} 38^{+} \mathrm{CD} 90^{+}\right)$ present in WT colon. Shown are representative plots from three independent experiments.

(B) Rspol mRNA expression in the indicated cell types sorted as described in panel (A). Data shown are normalized to Hprt expression level $(\mathrm{n}=4)$.

(C) Map3k2 mRNA expression in the indicated cell types sorted as described in panel (A). Data shown are normalized to Hprt expression level $(\mathrm{n}=4)$.

(D) Relative Rspol mRNA expression in $\mathrm{CD}_{326^{+}}$intestinal epithelial cells (IEC), CD $45^{+}$leukocytes, $\mathrm{CD}^{-} 0^{-}$intestinal mesenchymal stromal cells (CD45-CD31 $\left.{ }^{-} \mathrm{CD} 326^{-} \mathrm{gp} 38^{+} \mathrm{CD}^{-} 0^{-}\right)$, and $\mathrm{CD}^{+} 0^{+}$ intestinal mesenchymal stromal cells $\left(\mathrm{CD}^{\circ} 5^{-\mathrm{CD}} 31^{-} \mathrm{CD} 326^{-} \mathrm{gp} 38^{+} \mathrm{CD} 90^{\circ}\right)$ FACS-sorted from WT and Map3k2 deficient colons ( $\mathrm{n}=4$ /group) after 1 day treatment with 2\% DSS (or water only/untreated control). Data shown are normalized to Hprt expression level and are representative of two independent experiments.

(E) Bright-field images of matrigel-embedded colonic crypts cultured together with CD90 ${ }^{+}$ intestinal mesenchymal stromal cells in the presence of EGF, Noggin and Wnt3a for 3 days. CD90 ${ }^{+}$ intestinal mesenchymal stromal cells were sorted by flow cytometry from WT and Map $3 k 2^{-/}$mouse colons after 1-day DSS treatment (or water only/untreated control).

(F) Spheroids percentages among total colonoids derived after 3 days culture under the conditions described in panel (E) (n=3/group).

(G) Statistical analysis of average colonoid circumference after 3 days culture under the conditions 
described in panel $(\mathrm{E})$.

(H) Rspol mRNA detection using fluorescence in situ hybridization (FISH)-based RNAScope analysis of colons from WT and $M a p 3 k 2^{--}$mice after 1-day exposure to $2 \%$ DSS (or normal drinking water control). Dashed orange box inserts $(40 \mu \mathrm{m} * 80 \mu \mathrm{m})$ in each panel indicate the base (1), middle (2), and top (3) sections of the sub-epithelial area along the crypt axis.

(I) Quantification of Rspol mRNA expression by FISH (RNAScope) as described in panel (G). Fluorescence signals were counted within defined box areas covering 15 individual sub-epithelial regions along the crypt axis from base to top. Shown are summary data of Rspol mRNA fluorescence signal per $40 * 80 \mu^{2}$ area of the crypt axis for the base, middle and top regions. Error bars indicate mean \pm SEM $(* \mathrm{p}<0.05, * * \mathrm{p}<0.01, * * * \mathrm{p}<0.001$ by unpaired Student's $\mathrm{t}$ test).

\section{Figure 5. Map3k2-regulated Intestinal Stromal Cells are Defined by a CD90 ${ }^{\mathrm{mid}} \mathrm{CD81}^{+}$ \\ Phenotype}

(A) t-Distributed Stochastic Neighbor Embedding (t-SNE) plot of the scRNA-seq dataset generated by $\mathrm{CD} 45^{-} \mathrm{CD} 326^{-} \mathrm{CD} 31^{-} \mathrm{gp} 38^{+} \mathrm{CD} 90^{\mathrm{mid}}$ stromal cells isolated from WT mouse colon after 3 days exposure to $2 \%$ DSS in drinking water. Each dot represents an individual cell, color-coded according to cluster annotation.

(B) Violin plots of the indicated genes expressed in clusters 1, 2, 3, and 5.

(C) t-SNE plot showing Rspol gene expression in the scRNA-seq dataset at single cell level.

(D) Heat-map of genes that were significantly differentially expressed between cluster 2 and cluster 5, shown at single cell level.

(E) Violin plots of highly-ranked marker genes (non-TF) and transcriptional regulators (TF) expressed in cluster 2 and cluster 5, as compared with other clusters, as indicated.

(F) t-SNE plots showing $C d 81, C d 34$, and $S d c 1$ gene expression in the scRNA-seq dataset at single cell resolution.

(G) Representative flow cytometry plots showing percentage of cluster 5 stromal cells (CD45;,

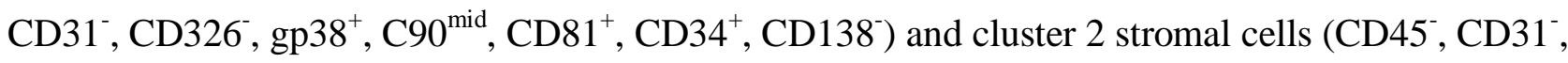




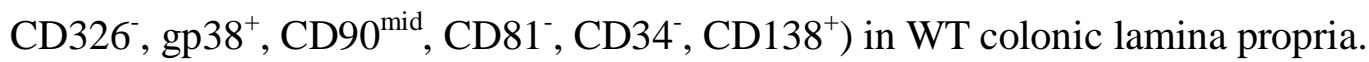

(H) Bar graph quantitation of the percentage of cluster 5 stromal cells (CD45, CD31, CD326, gp $\left.38^{+}, \mathrm{CD}^{\mathrm{mid}}, \mathrm{CD}^{+} 1^{+}, \mathrm{CD} 34^{+}, \mathrm{CD} 138^{-}\right)$in gp38 ${ }^{+}$stromal cells as assessed by flow cytometry analysis in WT and Map $3 k^{-/}$mouse colons (each $\mathrm{n}=3$ ) after treatment or not with DSS as indicated. Shown are representative plots from three independent experiments.

(I) Bar graph quantitation of the percentage of cluster 2 stromal cells (CD45, CD31, CD326;,

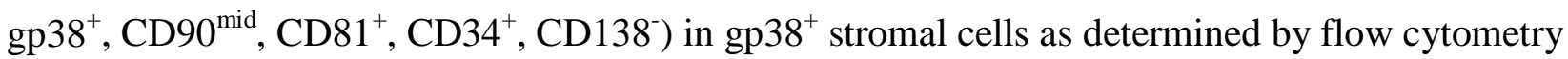
analysis in WT and Map $3 \mathrm{k}^{-/}$mouse colons (each $\mathrm{n}=3$ ) after 2 days exposure to DSS (or water only control) as indicated.

(J) Intestinal stromal cell clusters 2 and 5 were FACS-sorted from the colonic tissue of WT and Map $3 \mathrm{k}^{-/-}$mice (each $\mathrm{n}=3$ ) after 2 days exposure to DSS (or water only control) and total RNA was extracted for detection of Rspol mRNA. Data are normalized to Hprt expression level.

(K) Flow cytometry analysis of CD34 and CD81 expression in WT colonic lamina propria showing

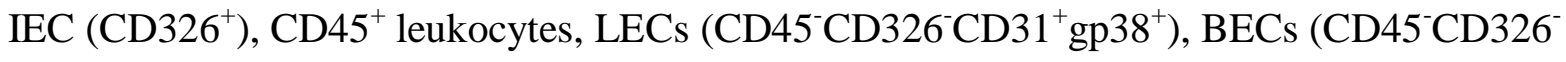

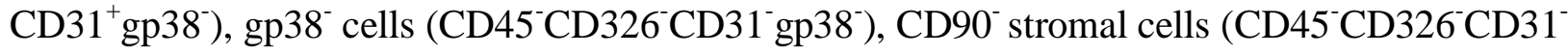
gp $\left.38^{+} \mathrm{CD}^{-} 0^{-}\right)$and $\mathrm{CD} 90^{\mathrm{mid}}$ stromal cells (CD45-CD326-CD31-gp $\left.38^{+} \mathrm{CD} 90^{\mathrm{mid}}\right)$. Plots are representative of three independent experiments.

(L) WT mouse colon was harvested and processed into swiss roll sections for staining with antiCD81 (red), anti-CD34 (green), anti-CD31 (blue) and then visualized using a Leica SP8 microscope with $63 x$ oil objective lens. The top row panels show the images of three separate channels as indicated. The left middle panel show an image of merged channels, and the center panel in the middle row shows a magnified image of the area from the left panel highlighted by dotted lines. The right panel in the middle row shows the CD81 and CD34 double positive staining marked with a pseudo-yellow color. The bottom row panels show CD81 and CD34 double positive cells (yellow) superimposed on each of the other channels as indicated. Pictures displayed are representative of two independent experiments.

Error bars indicate mean \pm SEM $(* \mathrm{p}<0.05, * * \mathrm{p}<0.01, * * * \mathrm{p}<0.001$ by unpaired Student's $\mathrm{t}$ test). 


\section{Figure 6. Map3k2 in Intestinal Stromal Cells Protects Mice from DSS-Induced Colitis in vivo} (A) qRT-PCR analysis of Map3k2 mRNA expression in flow cytometry-sorted CD $326^{+} \mathrm{CD} 45^{-}$ intestinal epithelial cells (IECs), $\mathrm{CD}^{+} 5^{+} \mathrm{CD} 326^{-}$leukocytes $\left(\mathrm{CD} 45^{+}\right), \mathrm{CD} 31^{+} \mathrm{CD} 45^{-} \mathrm{CD} 326^{-}$ endothelial cells $\left(\mathrm{CD} 31^{+}\right)$, and gp $38^{+} \mathrm{CD} 45^{\circ} \mathrm{CD} 31^{-} \mathrm{CD} 326^{-}$intestinal mesenchymal stromal cells $\left(\mathrm{gp} 38^{+}\right)$from the colons of tamoxifen treated Map3k $2^{f l f l}$ and Colla2-Cre $e^{E R T 2}:$ Map3 $k 2^{f l l f l}$ mice. Data presented are normalized to Hprt expression level and are representative of two independent experiments.

(B) Map $3 k^{f l l f l}$ and Colla2-Cre ${ }^{E R T 2}: M a p 3 k 2^{f l f l}$ co-housed littermate mice (n=3/group, 8 weeks old) were injected intraperitoneally with tamoxifen (TM) every two days ( $2 \mathrm{mg} / \mathrm{mouse} / \mathrm{time}, 5$ times). After 3 weeks from the last time of injection, mice were administered with $2 \%$ DSS in drinking water for 7 days followed by regular drinking water for additional 2 days. Body weights were recorded daily.

(C) Daily stool scores for mice treated as described in panel (B).

(D) Bar graphs show colon length of mice treated as described in panel (B) before sacrifice on day 9.

(E) Hematoxylin and eosin (H\&E) staining of colon (left panels) and histological assessment of the mucosa (right panels) in mice treated as described in panel (B) sacrificed on day 9.

(F) Colonic mRNA expression levels of Lgr5 (intestinal stem cell marker) and Il6 in mice treated as described in panel (B) sacrificed on day 9. Data shown are normalized to Hprt expression level. (G) Immunoblot analysis of MAP3K2 expression in intestinal epithelial cells (IEC) and intestinal mesenchymal stromal cells (IMSC) isolated from the colons of Map3k2 $2^{f l f l}$ and Vill-Cre:Map3k $2^{f l f l}$ mice. The GAPDH level was determined as loading controls.

(H) Map3k $2^{f f l}$ and Vill-Cre:Map $3 k 2^{f l f l}$ co-housed littermate mice (n=4 pairs) were administered with 2\% DSS in drinking water for 7 days followed by regular drinking water for additional 2 days. Body weights were recorded daily. Shown is a representative graph of two independent experiments. (I) Daily stool scores for mice treated as described in panel $(\mathrm{H})$. 
(J) Bar graphs show colon length of mice treated as described in panel $(\mathrm{H})$ before sacrifice on day 9. (K) Hematoxylin and eosin (H\&E) staining of colon (left panels) and histological assessment of the mucosa (right panels) in mice treated as described in panel $(\mathrm{H})$ before sacrifice on day 9.

(L) Colonic mRNA expression levels of Lgr5 and Il6 in mice treated as described in panel (H) before sacrifice on day 9. Data shown are normalized to Hprt expression level.

Error bars indicate mean $\pm \operatorname{SEM}(* \mathrm{p}<0.05$, $* * \mathrm{p}<0.01 * * * \mathrm{p}<0.001$ by unpaired Student's $\mathrm{t}$ test $)$. 


\section{References}

Amir el, A.D., Davis, K.L., Tadmor, M.D., Simonds, E.F., Levine, J.H., Bendall, S.C., Shenfeld, D.K., Krishnaswamy, S., Nolan, G.P., and Pe'er, D. (2013). viSNE enables visualization of high dimensional single-cell data and reveals phenotypic heterogeneity of leukemia. Nature biotechnology 31, 545-552.

Angerer, P., Haghverdi, L., Buttner, M., Theis, F.J., Marr, C., and Buettner, F. (2016). destiny: diffusion maps for largescale single-cell data in R. Bioinformatics 32, 1241-1243.

Aoki, R., Shoshkes-Carmel, M., Gao, N., Shin, S., May, C.L., Golson, M.L., Zahm, A.M., Ray, M., Wiser, C.L., Wright, C.V., et al. (2016). Foxl1-expressing mesenchymal cells constitute the intestinal stem cell niche. Cellular and molecular gastroenterology and hepatology 2, 175-188.

Barker, N., Bartfeld, S., and Clevers, H. (2010). Tissue-resident adult stem cell populations of rapidly self-renewing organs. Cell stem cell 7, 656-670.

Barker, N., van Es, J.H., Kuipers, J., Kujala, P., van den Born, M., Cozijnsen, M., Haegebarth, A., Korving, J., Begthel, H., Peters, P.J., et al. (2007). Identification of stem cells in small intestine and colon by marker gene Lgr5. Nature 449, 1003-1007.

Binnerts, M.E., Kim, K.A., Bright, J.M., Patel, S.M., Tran, K., Zhou, M., Leung, J.M., Liu, Y., Lomas, W.E., 3rd, Dixon, M., et al. (2007). R-Spondin1 regulates Wnt signaling by inhibiting internalization of LRP6. Proceedings of the National Academy of Sciences of the United States of America 104, 14700-14705.

Butler, A., Hoffman, P., Smibert, P., Papalexi, E., and Satija, R. (2018). Integrating single-cell transcriptomic data across different conditions, technologies, and species. Nature biotechnology 36, 411-420.

Chang, X., Liu, F., Wang, X., Lin, A., Zhao, H., and Su, B. (2011). The kinases MEKK2 and MEKK3 regulate transforming growth factor-beta-mediated helper T cell differentiation. Immunity 34, 201-212.

Cheng, J., Yang, J., Xia, Y., Karin, M., and Su, B. (2000). Synergistic interaction of MEK kinase 2, C-Jun N-terminal kinase (JNK) kinase 2, and JNK1 results in efficient and specific JNK1 activation. Molecular and cellular biology 20, $2334-2342$. Cheng, J., Zhang, D., Kim, K., Zhao, Y., Zhao, Y., and Su, B. (2005). Mip1, an MEKK2-interacting protein, controls MEKK2 dimerization and activation. Molecular and cellular biology 25, 5955-5964.

de Lau, W., Peng, W.C., Gros, P., and Clevers, H. (2014). The R-spondin/Lgr5/Rnf43 module: regulator of Wnt signal strength. Genes \& development 28, 305-316.

Degirmenci, B., Valenta, T., Dimitrieva, S., Hausmann, G., and Basler, K. (2018). GLI1-expressing mesenchymal cells form the essential Wnt-secreting niche for colon stem cells. Nature 558, 449-453.

Elinav, E., Strowig, T., Kau, A.L., Henao-Mejia, J., Thaiss, C.A., Booth, C.J., Peaper, D.R., Bertin, J., Eisenbarth, S.C., Gordon, J.I., et al. (2011). NLRP6 inflammasome regulates colonic microbial ecology and risk for colitis. Cell 145, 745757.

Greenblatt, M.B., Shin, D.Y., Oh, H., Lee, K.Y., Zhai, B., Gygi, S.P., Lotinun, S., Baron, R., Liu, D., Su, B., et al. (2016). MEKK2 mediates an alternative beta-catenin pathway that promotes bone formation. Proceedings of the National Academy of Sciences of the United States of America 113, E1226-1235.

Greicius, G., Kabiri, Z., Sigmundsson, K., Liang, C., Bunte, R., Singh, M.K., and Virshup, D.M. (2018). PDGFRalpha(+) pericryptal stromal cells are the critical source of Wnts and RSPO3 for murine intestinal stem cells in vivo. Proceedings of the National Academy of Sciences of the United States of America 115, E3173-E3181.

Guo, Z., Clydesdale, G., Cheng, J., Kim, K., Gan, L., McConkey, D.J., Ullrich, S.E., Zhuang, Y., and Su, B. (2002). Disruption of Mekk2 in mice reveals an unexpected role for MEKK2 in modulating T-cell receptor signal transduction. Molecular and cellular biology 22, 5761-5768. 
Haber, A.L., Biton, M., Rogel, N., Herbst, R.H., Shekhar, K., Smillie, C., Burgin, G., Delorey, T.M., Howitt, M.R., Katz, Y., et al. (2017). A single-cell survey of the small intestinal epithelium. Nature 551, 333-339.

Han, X., Wang, R., Zhou, Y., Fei, L., Sun, H., Lai, S., Saadatpour, A., Zhou, Z., Chen, H., Ye, F., et al. (2018). Mapping the Mouse Cell Atlas by Microwell-Seq. Cell 172, 1091-1107 e1017.

Huynh, P.T., Beswick, E.J., Coronado, Y.A., Johnson, P., O'Connell, M.R., Watts, T., Singh, P., Qiu, S., Morris, K., Powell, D.W., et al. (2016). CD90(+) stromal cells are the major source of IL-6, which supports cancer stem-like cells and inflammation in colorectal cancer. International journal of cancer 138, 1971-1981.

Kabiri, Z., Greicius, G., Madan, B., Biechele, S., Zhong, Z., Zaribafzadeh, H., Edison, Aliyev, J., Wu, Y., Bunte, R., et al. (2014). Stroma provides an intestinal stem cell niche in the absence of epithelial Wnts. Development 141, $2206-2215$. Kang, S.H., Fukaya, M., Yang, J.K., Rothstein, J.D., and Bergles, D.E. (2010). NG2+ CNS glial progenitors remain committed to the oligodendrocyte lineage in postnatal life and following neurodegeneration. Neuron 68, 668-681. Karpus, O.N., Westendorp, B.F., Vermeulen, J.L.M., Meisner, S., Koster, J., Muncan, V., Wildenberg, M.E., and van den Brink, G.R. (2019). Colonic CD90+ Crypt Fibroblasts Secrete Semaphorins to Support Epithelial Growth. Cell reports 26, 3698-3708.e3695.

Kim, K.A., Kakitani, M., Zhao, J., Oshima, T., Tang, T., Binnerts, M., Liu, Y., Boyle, B., Park, E., Emtage, P., et al. (2005). Mitogenic influence of human R-spondin1 on the intestinal epithelium. Science 309, 1256-1259.

Kinchen, J., Chen, H.H., Parikh, K., Antanaviciute, A., Jagielowicz, M., Fawkner-Corbett, D., Ashley, N., Cubitt, L., Mellado-Gomez, E., Attar, M., et al. (2018). Structural Remodeling of the Human Colonic Mesenchyme in Inflammatory Bowel Disease. Cell 175, 372-386 e317.

Koch, S. (2017). Extrinsic control of Wnt signaling in the intestine. Differentiation; research in biological diversity $97,1-$ 8.

Kretzschmar, K., and Clevers, H. (2017). Wnt/beta-catenin signaling in adult mammalian epithelial stem cells.

Developmental biology 428, 273-282.

Madison, B.B., Dunbar, L., Qiao, X.T.T., Braunstein, K., Braunstein, E., and Gumucio, D.L. (2002). cis elements of the villin gene control expression in restricted domains of the vertical (crypt) and horizontal (duodenum, cecum) axes of the intestine. Journal of Biological Chemistry 277, 33275-33283.

Messina, V., Buccione, C., Marotta, G., Ziccheddu, G., Signore, M., Mattia, G., Puglisi, R., Sacchetti, B., Biancone, L., and Valtieri, M. (2017). Gut Mesenchymal Stromal Cells in Immunity. Stem cells international 2017, 8482326.

Mifflin, R.C., Pinchuk, I.V., Saada, J.I., and Powell, D.W. (2011). Intestinal myofibroblasts: targets for stem cell therapy. American journal of physiology Gastrointestinal and liver physiology 300, G684-696.

Nanki, K., Toshimitsu, K., Takano, A., Fujii, M., Shimokawa, M., Ohta, Y., Matano, M., Seino, T., Nishikori, S., Ishikawa, K., et al. (2018). Divergent Routes toward Wnt and R-spondin Niche Independency during Human Gastric Carcinogenesis. Cell 174, 856-869 e817.

Ootani, A., Li, X., Sangiorgi, E., Ho, Q.T., Ueno, H., Toda, S., Sugihara, H., Fujimoto, K., Weissman, I.L., Capecchi, M.R., et al. (2009). Sustained in vitro intestinal epithelial culture within a Wnt-dependent stem cell niche. Nature medicine 15, 701-706.

Owens, B.M., and Simmons, A. (2013). Intestinal stromal cells in mucosal immunity and homeostasis. Mucosal immunology 6, 224-234.

Pinchuk, I.V., Mifflin, R.C., Saada, J.I., and Powell, D.W. (2010). Intestinal mesenchymal cells. Current gastroenterology reports $12,310-318$.

Pinto, D., Gregorieff, A., Begthel, H., and Clevers, H. (2003). Canonical Wnt signals are essential for homeostasis of the 
intestinal epithelium. Genes \& development 17, 1709-1713.

Powell, D.W., Pinchuk, I.V., Saada, J.I., Chen, X., and Mifflin, R.C. (2011). Mesenchymal cells of the intestinal lamina propria. Annual review of physiology 73, 213-237.

Rinkevich, Y., Mori, T., Sahoo, D., Xu, P.X., Bermingham, J.R., Jr., and Weissman, I.L. (2012). Identification and prospective isolation of a mesothelial precursor lineage giving rise to smooth muscle cells and fibroblasts for mammalian internal organs, and their vasculature. Nature cell biology 14, 1251-1260.

Roulis, M., and Flavell, R.A. (2016). Fibroblasts and myofibroblasts of the intestinal lamina propria in physiology and disease. Differentiation; research in biological diversity 92, 116-131.

Roulis, M., Nikolaou, C., Kotsaki, E., Kaffe, E., Karagianni, N., Koliaraki, V., Salpea, K., Ragoussis, J., Aidinis, V., Martini, E., et al. (2014). Intestinal myofibroblast-specific Tpl2-Cox-2-PGE2 pathway links innate sensing to epithelial homeostasis. Proceedings of the National Academy of Sciences of the United States of America 111, E4658-4667.

Sato, T., van Es, J.H., Snippert, H.J., Stange, D.E., Vries, R.G., van den Born, M., Barker, N., Shroyer, N.F., van de Wetering, M., and Clevers, H. (2011). Paneth cells constitute the niche for Lgr 5 stem cells in intestinal crypts. Nature 469, 415-418. Sato, T., Vries, R.G., Snippert, H.J., van de Wetering, M., Barker, N., Stange, D.E., van Es, J.H., Abo, A., Kujala, P., Peters, P.J., et al. (2009). Single Lgr5 stem cells build crypt-villus structures in vitro without a mesenchymal niche. Nature 459, 262-265.

Sawada, S., Scarborough, J.D., Killeen, N., and Littman, D.R. (1994). A lineage-specific transcriptional silencer regulates CD4 gene expression during T lymphocyte development. Cell 77, 917-929.

Schuijers, J., Junker, J.P., Mokry, M., Hatzis, P., Koo, B.K., Sasselli, V., van der Flier, L.G., Cuppen, E., van Oudenaarden, A., and Clevers, H. (2015). Ascl2 acts as an R-spondin/Wnt-responsive switch to control stemness in intestinal crypts. Cell stem cell 16, 158-170.

Shoshkes-Carmel, M., Wang, Y.J., Wangensteen, K.J., Toth, B., Kondo, A., Massasa, E.E., Itzkovitz, S., and Kaestner, K.H. (2018). Subepithelial telocytes are an important source of Wnts that supports intestinal crypts. Nature 557, 242-246. Stzepourginski, I., Nigro, G., Jacob, J.M., Dulauroy, S., Sansonetti, P.J., Eberl, G., and Peduto, L. (2017). CD34+ mesenchymal cells are a major component of the intestinal stem cells niche at homeostasis and after injury. Proceedings of the National Academy of Sciences of the United States of America 114, E506-E513.

Su, B., Cheng, J., Yang, J., and Guo, Z. (2001). MEKK2 is required for T-cell receptor signals in JNK activation and interleukin-2 gene expression. The Journal of biological chemistry 276, 14784-14790.

Sun, W., Kesavan, K., Schaefer, B.C., Garrington, T.P., Ware, M., Johnson, N.L., Gelfand, E.W., and Johnson, G.L. (2001). MEKK2 associates with the adapter protein Lad/RIBP and regulates the MEK5-BMK1/ERK5 pathway. The Journal of biological chemistry 276, 5093-5100.

Takeda, N., Jain, R., LeBoeuf, M.R., Wang, Q., Lu, M.M., and Epstein, J.A. (2011). Interconversion between intestinal stem cell populations in distinct niches. Science 334, 1420-1424.

Thomson, C.A., van de Pavert, S.A., Stakenborg, M., Labeeuw, E., Matteoli, G., Mowat, A.M., and Nibbs, R.J.B. (2018). Expression of the Atypical Chemokine Receptor ACKR4 Identifies a Novel Population of Intestinal Submucosal Fibroblasts That Preferentially Expresses Endothelial Cell Regulators. Journal of immunology 201, 215-229.

Tsioumpekou, M., Papadopoulos, N., Burovic, F., Heldin, C.H., and Lennartsson, J. (2016). Platelet-derived growth factor (PDGF)-induced activation of Erk5 MAP-kinase is dependent on Mekk2, Mek1/2, PKC and PI3-kinase, and affects BMP signaling. Cellular signalling 28, 1422-1431.

Valenta, T., Degirmenci, B., Moor, A.E., Herr, P., Zimmerli, D., Moor, M.B., Hausmann, G., Cantu, C., Aguet, M., and Basler, K. (2016). Wnt Ligands Secreted by Subepithelial Mesenchymal Cells Are Essential for the Survival of Intestinal 
Stem Cells and Gut Homeostasis. Cell reports 15, 911-918.

Yan, K.S., Janda, C.Y., Chang, J., Zheng, G.X.Y., Larkin, K.A., Luca, V.C., Chia, L.A., Mah, A.T., Han, A., Terry, J.M., et al. (2017). Non-equivalence of Wnt and R-spondin ligands during Lgr5(+) intestinal stem-cell self-renewal. Nature 545, 238-242.

Zhang, D., Facchinetti, V., Wang, X., Huang, Q., Qin, J., and Su, B. (2006). Identification of MEKK2/3 serine phosphorylation site targeted by the Toll-like receptor and stress pathways. The EMBO journal 25, 97-107. Zhao, J., de Vera, J., Narushima, S., Beck, E.X., Palencia, S., Shinkawa, P., Kim, K.A., Liu, Y., Levy, M.D., Berg, D.J., et al. (2007). R-spondin1, a novel intestinotrophic mitogen, ameliorates experimental colitis in mice. Gastroenterology 132, 1331-1343.

Zhou, W.J., Geng, Z.H., Spence, J.R., and Geng, J.G. (2013). Induction of intestinal stem cells by R-spondin 1 and Slit2 augments chemoradioprotection. Nature 501, 107-111. 

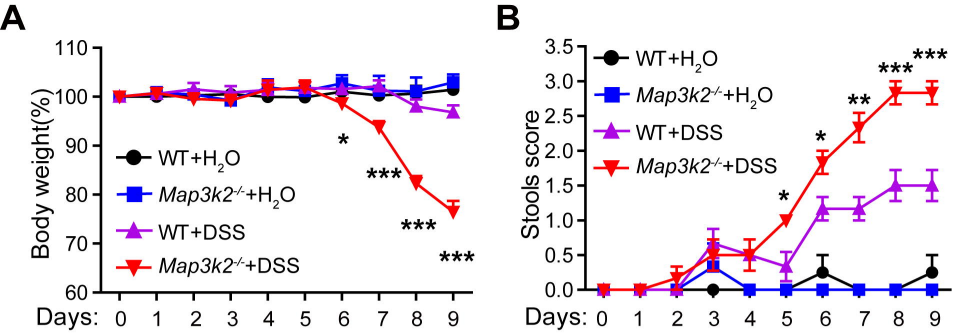

C
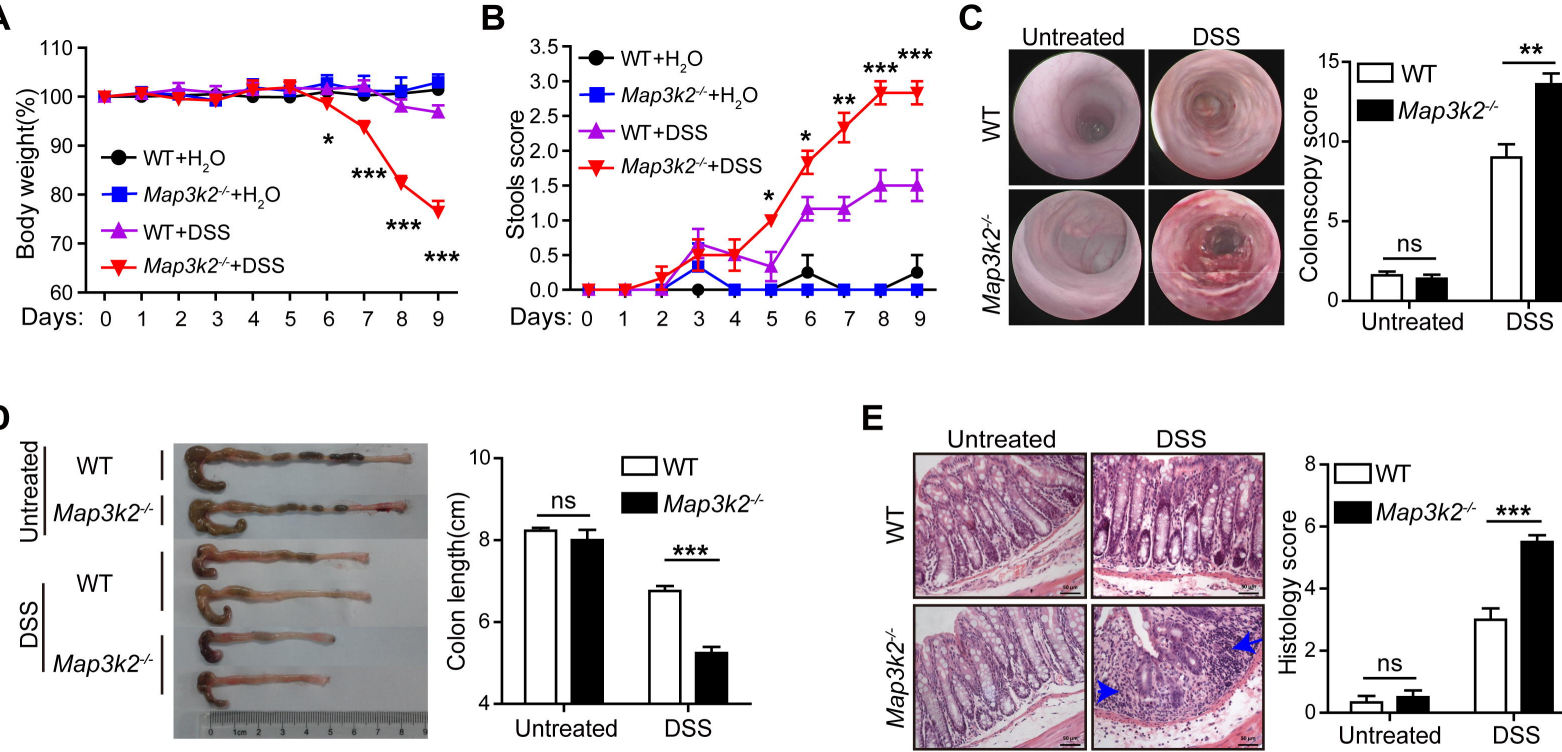

$\mathbf{F}$

.
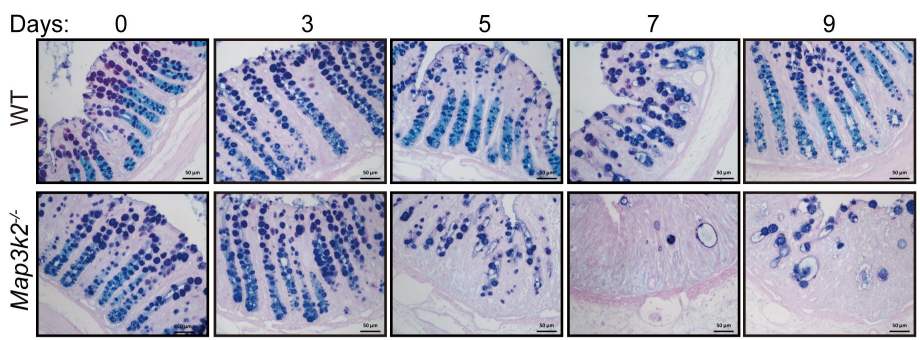

\section{$\square$ WT}

- Map3k2--

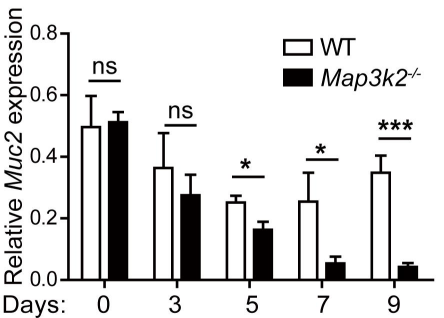

G

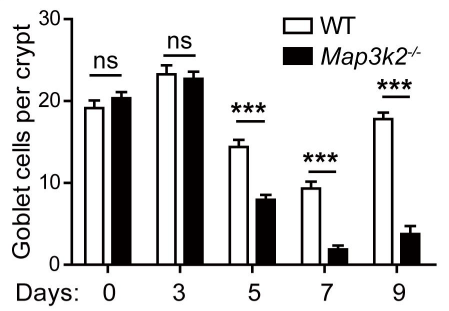




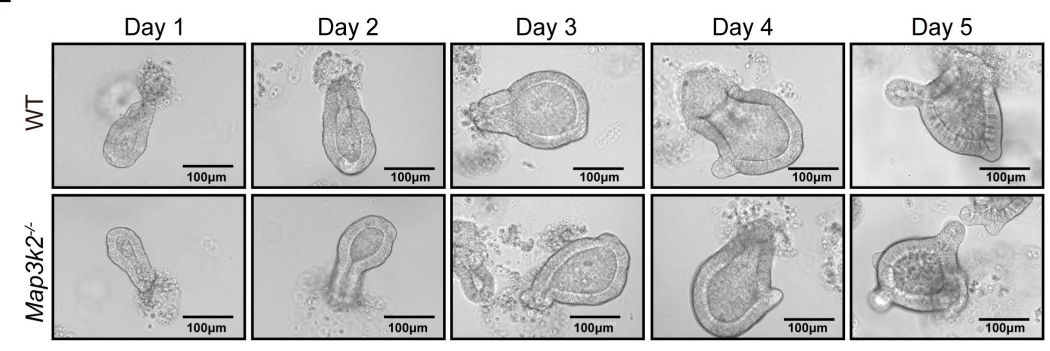

G

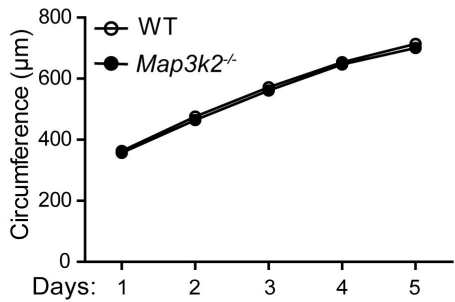

H
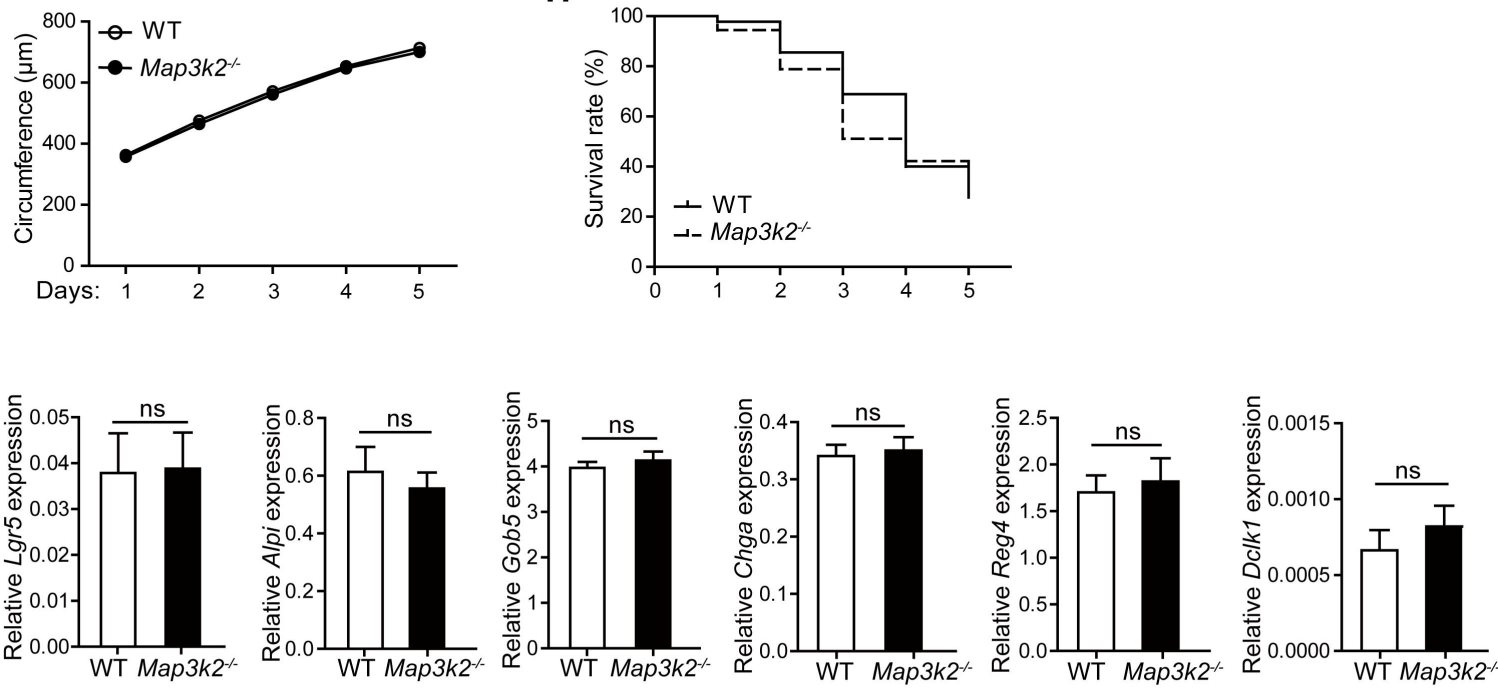

Figures 2F-2I 

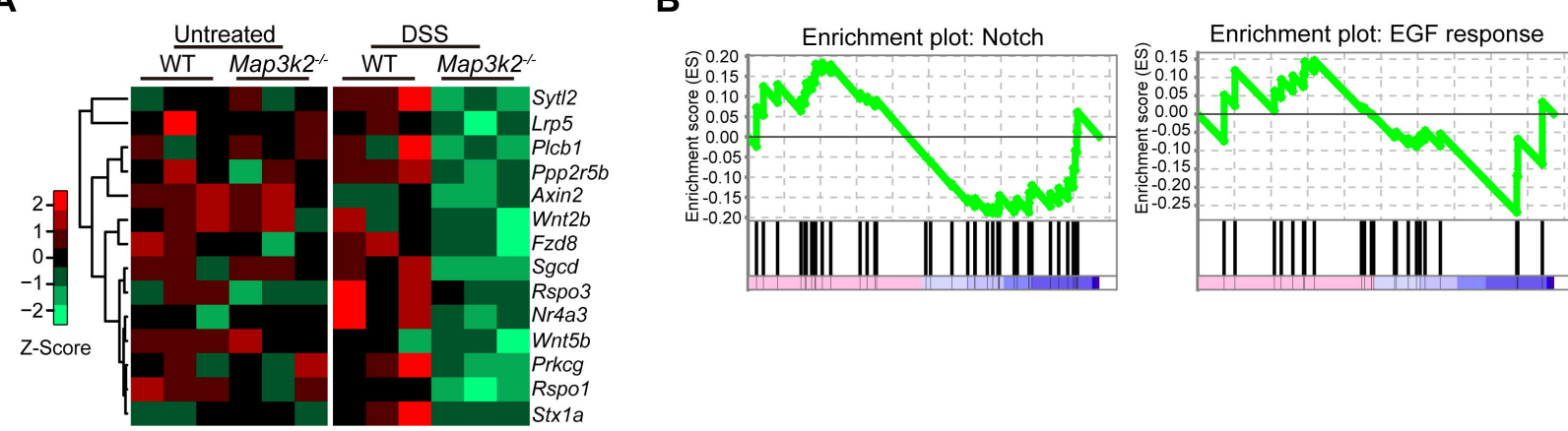

C
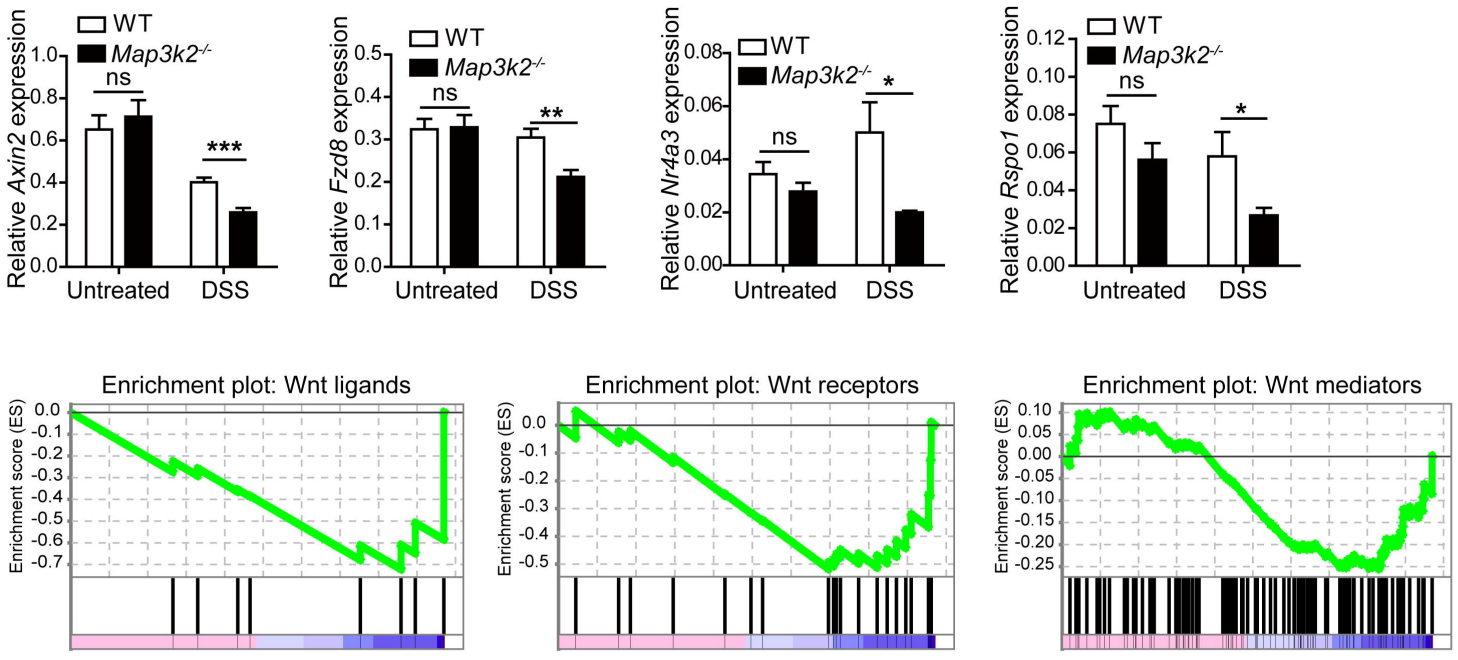

E

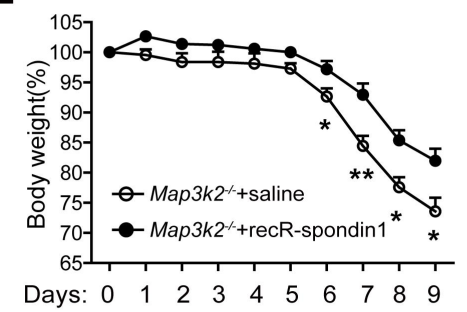

F

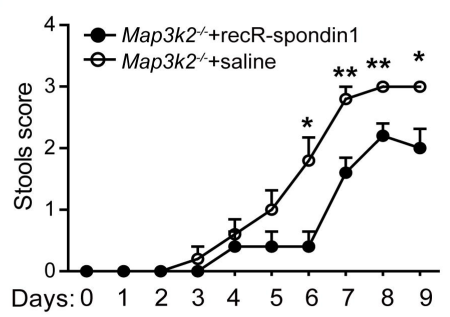

G
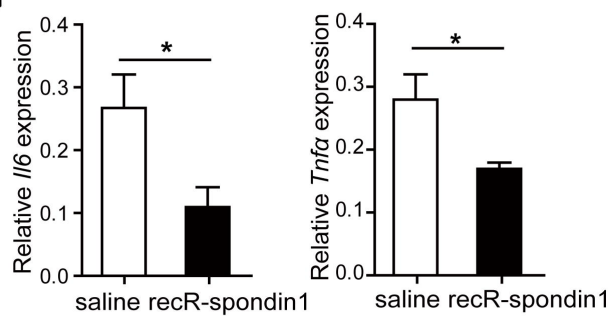

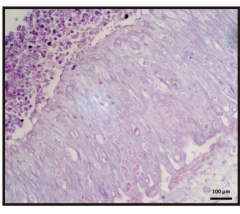

saline

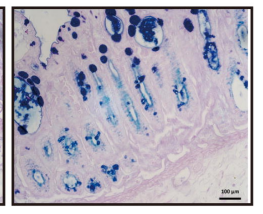

recR-spondin1

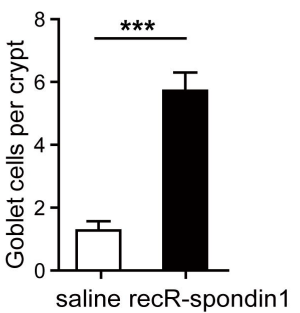

I

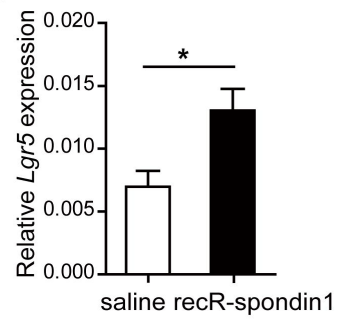




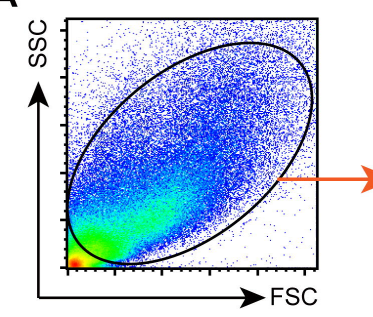

B

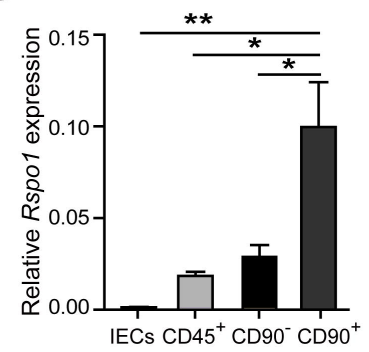

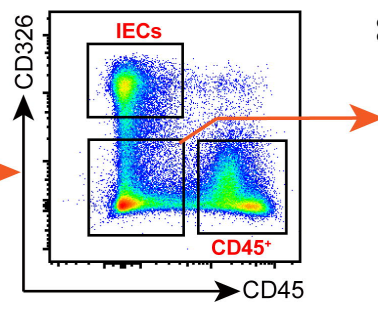

C

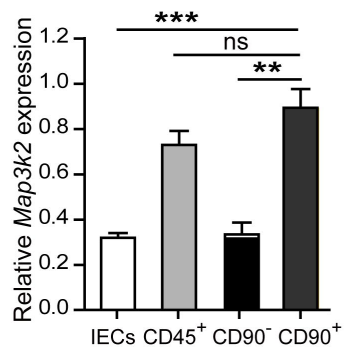

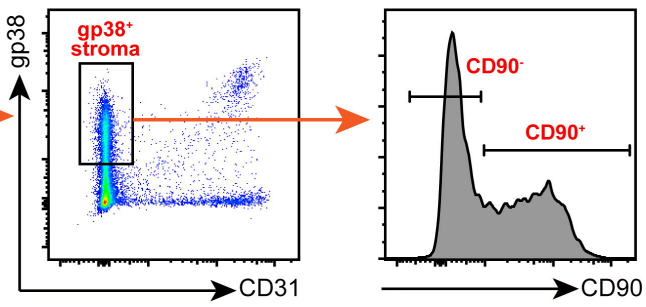

D

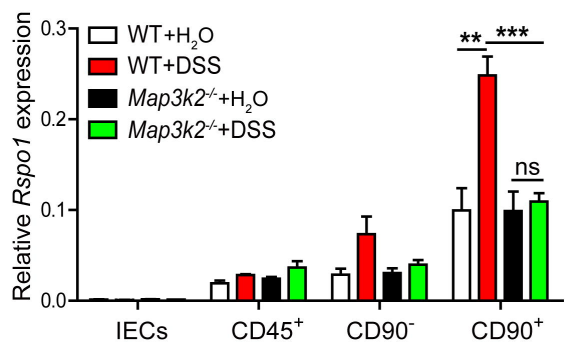

G
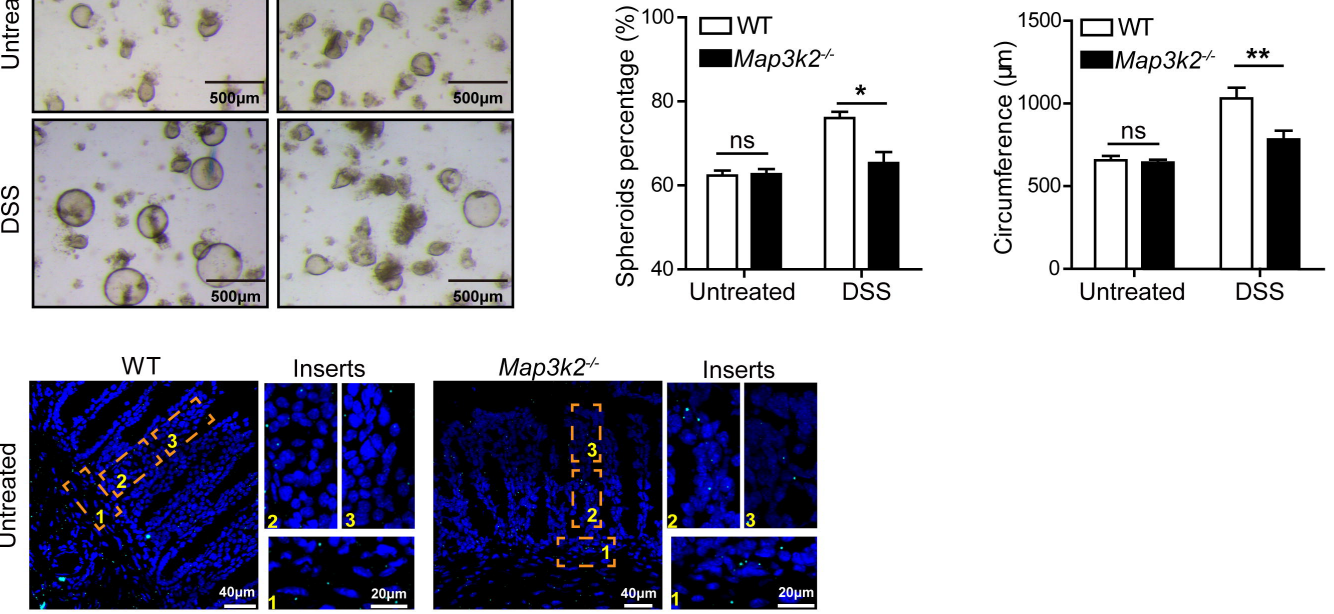

.
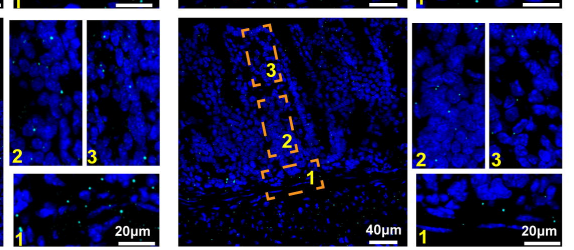

DAPI: Blue, Rspo1: Green dots, 1: Base, 2: Middle, 3: Top.
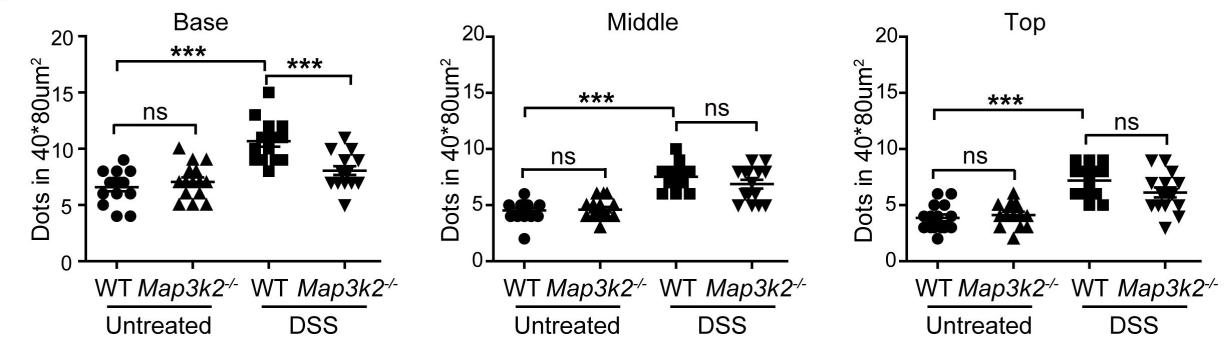

Figures $4 A-4$ 


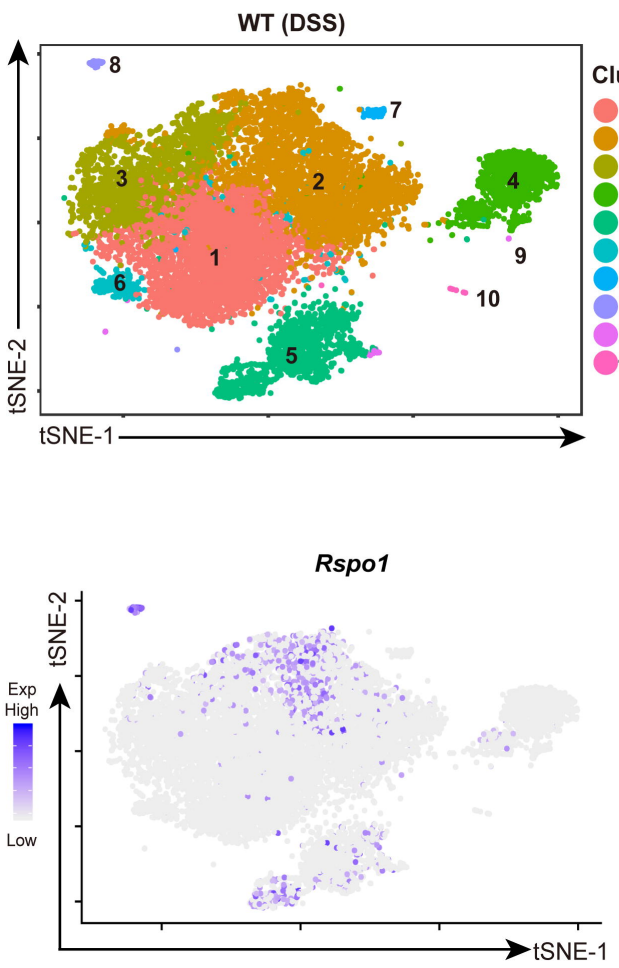

E

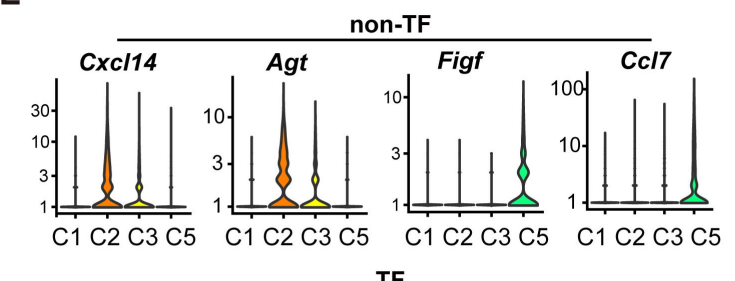

TF

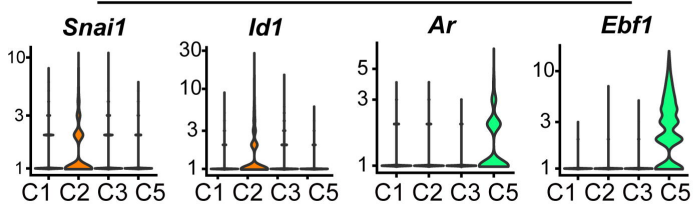

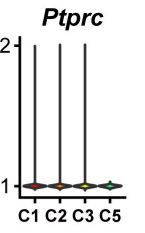
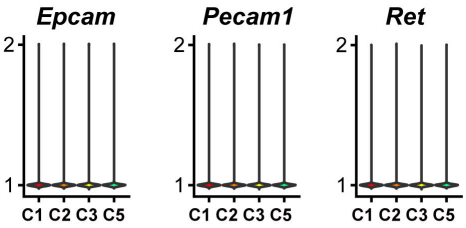

$6 \mathrm{MHCII}^{+}$stromal cells

7 Telocytes

8 Mesothelial cells

9 Interstitial Cell of Cajal

10 Epithelial cells
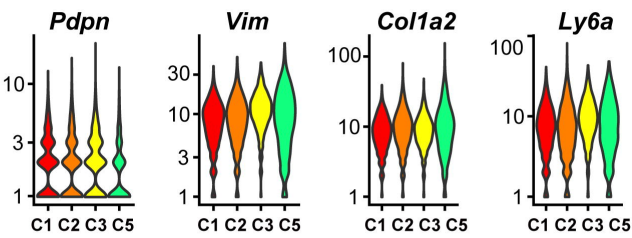

D

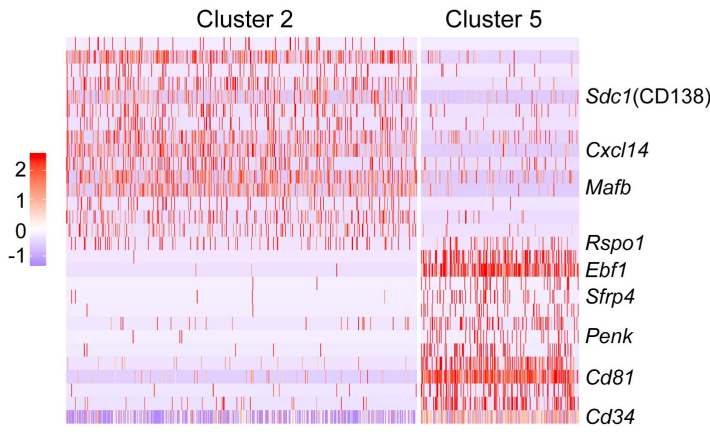

F

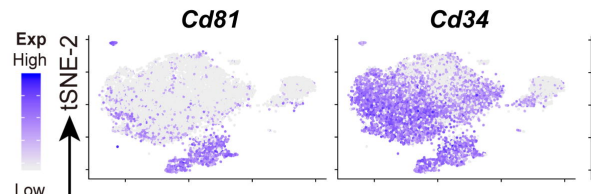

Sdc1 (CD138) 


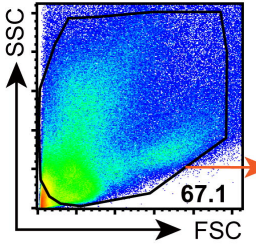

H

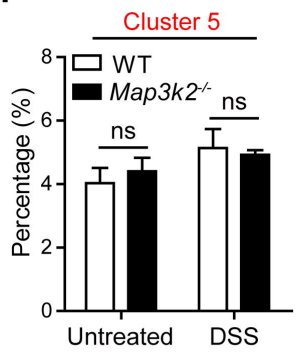

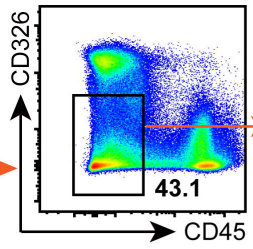
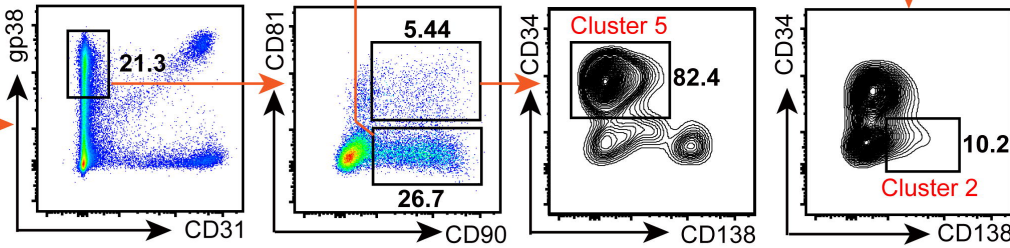

I

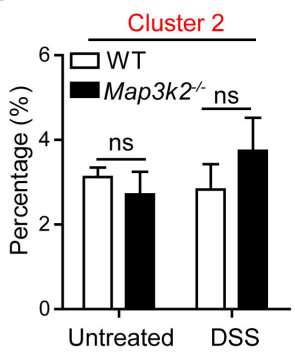

J

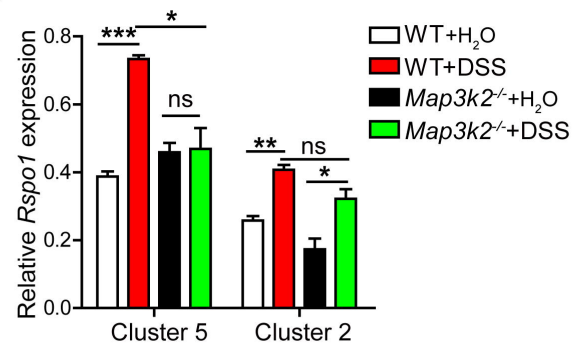

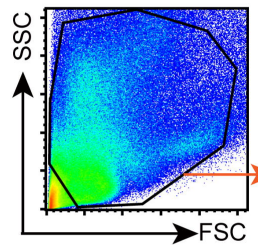
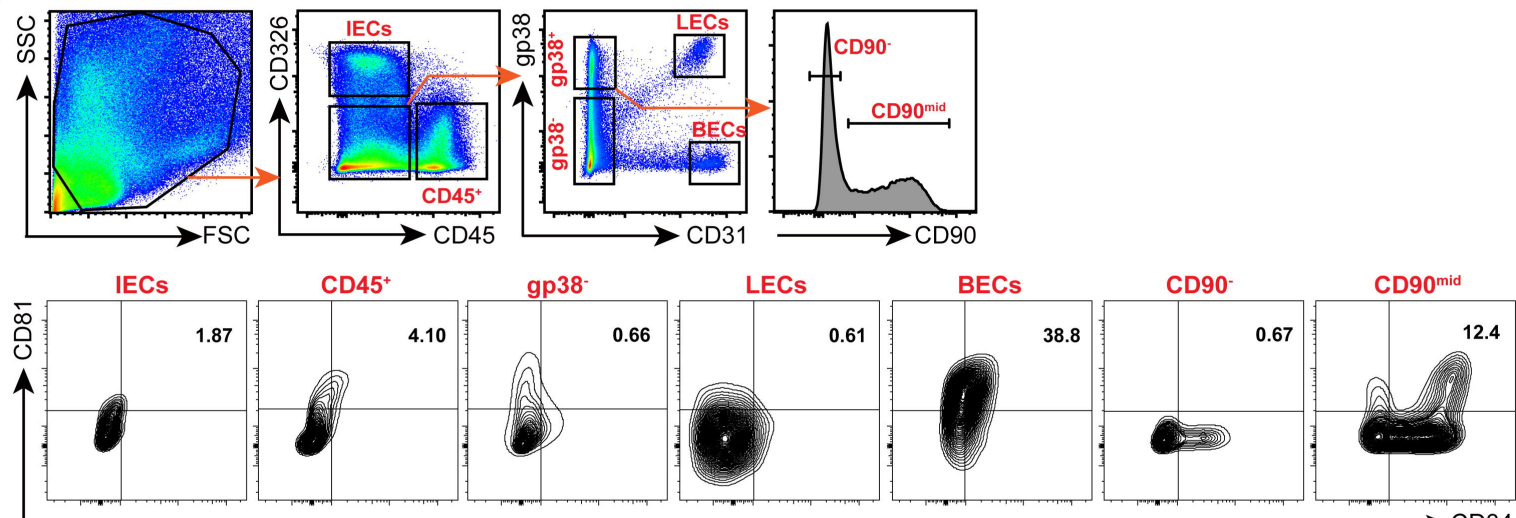

L
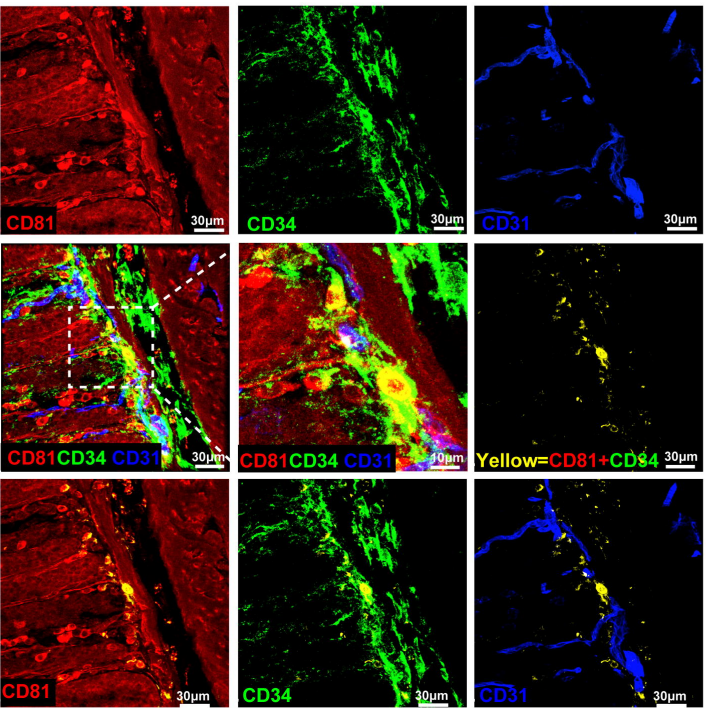

Figures 5G-5L 

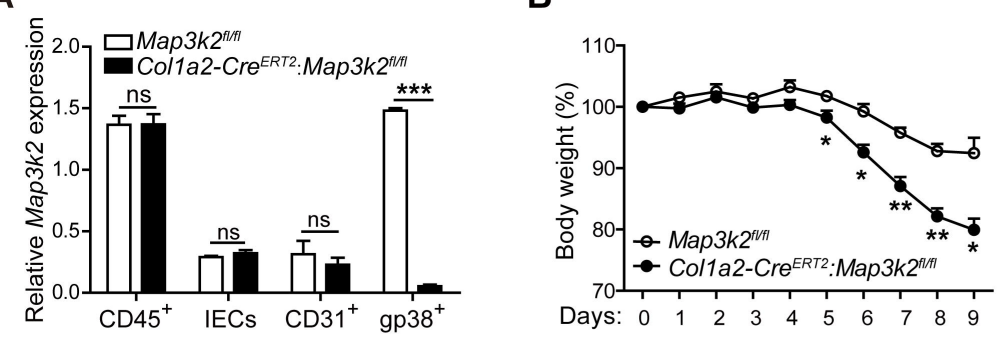

C

D

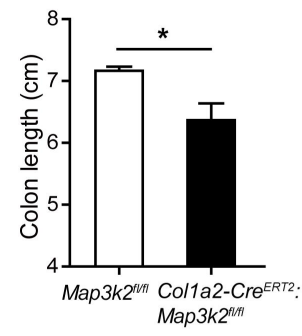

E

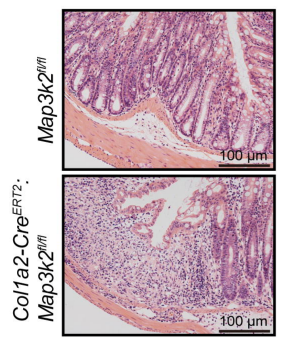

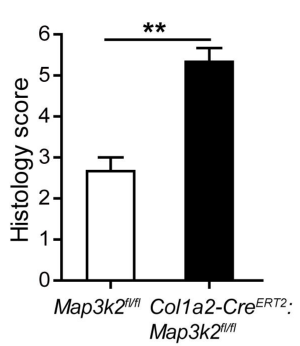

G

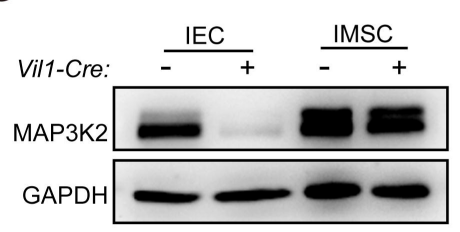

H

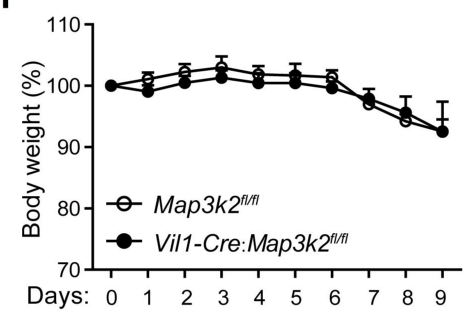

F
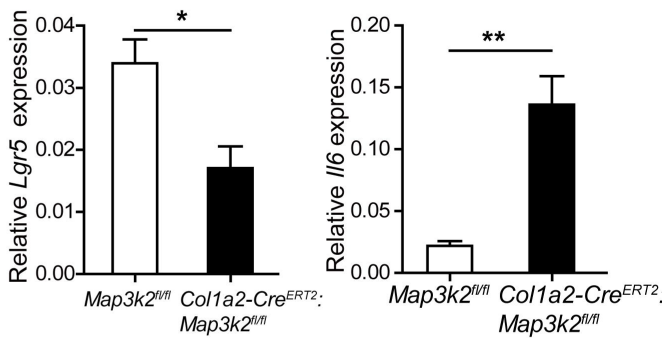

I

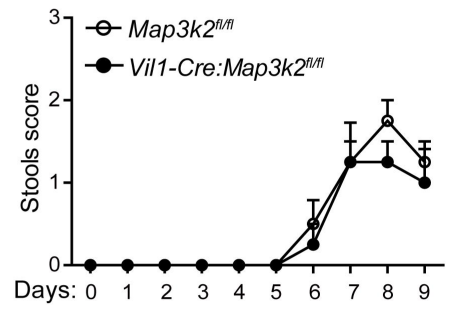

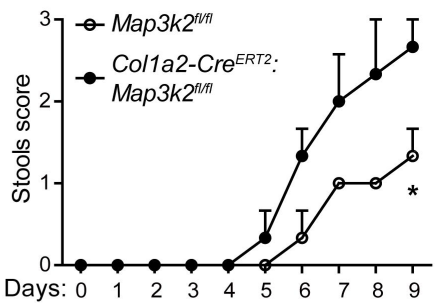

L

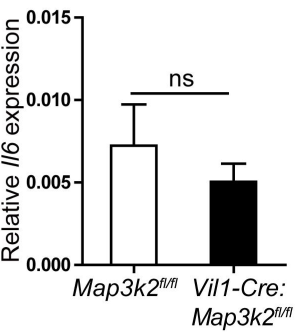

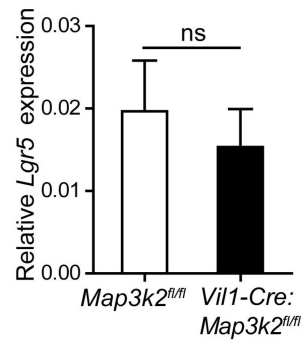

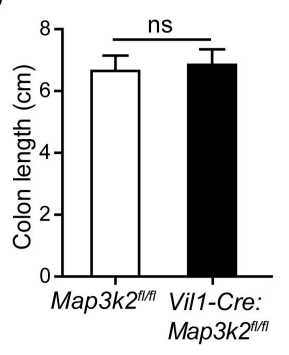

K

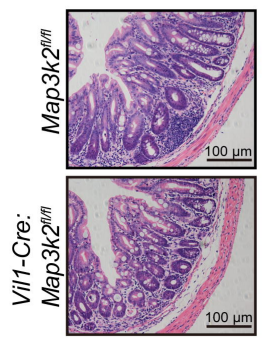

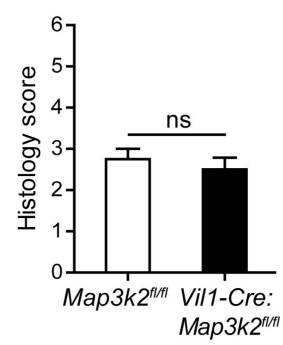

